\title{
Role of Chemical Kinetics in the Heterogeneous Catalysis Studies
}

\author{
L. A. PETROV*, Y. ALHAMED, A. AL-ZAHRANI, M. DAOUS \\ SABIC Chair in Catalysis, King Abdulaziz University, P.O. Box 80204, Jeddah 21589, Kingdom of Saudi Arabia
}

\begin{abstract}
The paper presents shortly some of the important elements of the theory and of the practical applications of the kinetics of heterogeneous catalytic reactions. Discussed are some of the most important concepts of the kinetics of complex heterogeneous catalytic reactions, methodology of building kinetic models and mathematical treatment of experimental data, influence of heat and mass transfer, types of laboratory reactors, kinetics and nanosized catalysts and others. Examples for use of the kinetic studies for the development and application of industrial catalysts and modeling of industrial reactors are presented.
\end{abstract}

Key words: kinetics; heterogeneous catalytic reaction; mass action; surface action; reaction rate; reaction mechanism

CLC number: O643 Document code: A

Received 7 February 2011. Accepted 23 March 2011.

*Corresponding author. Tel: +966-507589382; Fax:+966-2-69522571; E-mail: lpetrov@kau.edu.sa

This work was supported by the SABIC Chair in Catalysis, King Abdulaziz University.

English edition available online at Elsevier ScienceDirect (http://www.sciencedirect.com/science/journal/18722067).

Catalysis, as one of the most frequently observed natural phenomena, is the basic form of proceeding of chemical and biochemical reactions at a high rate in nature as well as in different fields of practical applications.

Being fundamental science, catalysis is developing at the interface of chemistry, physics, biology, and mathematics. This circumstance determines the great complexity and value of the investigations as well as the difficulty in interpretation of the obtained results.

Catalysis is the most powerful method of controlling and directing chemical reactions. Competent international forecasts place catalysis among the most promising fields of basic research. The successes of basic research in the field of catalysis have a direct effect on solving many fundamental, technological, environmental, and social problems that face humanity. These include efficient utilization of raw materials, invention of new materials and substances with pre-programmed properties and pharmaceutical products, development of efficient systems for environmental protection, introducing of new and renewable sources of energy and development of new processes and technologies in chemistry and biology. Catalytic processes form the fundamentals of modern chemical and petrochemical industries. Over $80 \%$ of the processes in these industries are catalytic, while for the newly introduced ones this percentage is over $90 \%$. In the highly developed industrial countries like USA, Japan, and Germany, catalytic processes create about $20 \%$ of the gross domestic product.

Catalysis is one of the top priority issues of chemical sciences of many research institutions, universities, and industrial companies [1]. The studies in the field of kinetics of heterogeneous catalytic reactions are an indispensable step of the theoretical and applied investigations on catalysis. They serve as fundamentals upon elucidation of the mechanism of a given heterogeneous catalytic reaction and contribute essentially to the elucidation of the catalyst behavior in the course of its preparation, exploitation, and regeneration. They serve as basis during modeling and selection of optimal catalysts and optimization of catalytic reactors. For these reasons, the development of theory and practice in catalysis is inconceivable without unfolding extensive kinetic investigations.

\section{Important topics in the studies of the kinetics heterogeneous catalytic reaction}

Chemical kinetics is a science studying the reaction rates of chemical reactions taking into account their reaction mechanism. Chemical kinetics is the basis of catalysis; however, catalysis is not a part of the kinetics.

The tasks that face now the researchers in the field of catalytic reaction kinetics could be formulated as follows.

Development of the theory of kinetics. The investigations in this direction give impetus to theoretical aspects of the kinetics, the methods of constructing kinetic models, the methods of evaluation of kinetic parameters, and the mathematical problems, connected with it.

Studies of the kinetics and mechanism of important in theoretical aspect catalytic processes. These studies aim at assisting the development of theory in catalysis, the selection 
of new catalytic systems and catalysts, elucidation of the intimate mechanism of the catalytic act, the nature of the active site, the behavior of the catalyst-reagent system as a whole.

Creation of kinetic models of industrially important reactions. The kinetic models of catalytic processes are the basis, on which the modeling and the design of catalytic reactors is carried out. The transition from molecular scale to macro scale during the practical realization of a given catalytic process is accomplished on this basis. That is why it is necessary that the kinetic model be able to reflect all essential aspects of the mechanism of the process.

Development of kinetic models of non-stationary catalytic processes. These models account for the following factors: the rates of the elementary chemical transformations, the rate of changing the composition and the activity of the catalyst, diffusion of the species in the catalyst, transfer of reactants from one type of active sites to others etc. The investigation of the dynamics of the catalytic process i.e. the evolution of the heterogeneous catalytic reactions is included in this set of tasks. It is on these results that the development of the studies on the dynamics and stability of the catalytic reactors is depending, which in their turn determine the optimal starting and operation conditions.

The studies of the kinetics of processes, occurring during the preparation, exploitation, deactivation, and regeneration of the industrial catalysts. In the course of preparation, operation and regeneration of the catalysts a series of processes are proceeding in them, which lead to alteration of their properties. The good knowledge and control of this type of alteration may bring considerable improvement of the quality of the catalysts and optimization of the regimes of their use.

The elaboration of theoretically grounded methods for testing and controlling the catalytic activity and selectivity of the industrial catalysts. Due to the strongly specific effect of the catalysts, it is not possible to propose universal methods for testing and controlling their catalytic activity and selectivity. These methods are individual for each catalytic system. For this reason the elaboration, unification, and standardization of these methods has great importance for the correct selection and evaluation of the studied catalysts.

Theory and automation of the kinetic experiments. The experimental studies in the field of chemical kinetics are time consuming, expensive, and demanding a large volume of research work. They require especially high degree of experience in order to set the task correctly and to select the proper experimental design. The dynamic development of the industrial catalysis puts on the agenda the problem to increase essentially the rate of kinetic model construction. The solving of this problem is unthinkable without applying the achievements of the optimal experiment theory, the use of automated systems for carrying out the kinetic experiments and data acquisition on the basis of modern computers during all stages of obtaining and processing the experimental data. The recently introduced high throughput experiments approach is proved very effective in many areas of catalysis studies.

\section{Elements of kinetics of complex heterogeneous catalytic reactions}

A comprehensive description of the theory of kinetics of complex heterogeneous catalytic reactions developed by Horiuti and Temkin can be found in Refs. [2-4]. Several excellent books on catalysis were published recently [5-11] where some information about this theory is presented. Here we will give very condensed description of the basic concepts of this theory.

From the point of view of the complexity of the composition of a reaction mixture and the number of reactions taking place simultaneously, the catalytic processes under study can be divided into two groups: single route catalytic processes and multiroute catalytic processes.

Single route catalytic processes. The stoichiometry of the single route catalytic processes is described by one stoichiometric equation. This group includes most of petrochemical processes (hydrogenation processes).

Multiroute catalytic processes. We shall call multiroute (complex) heterogeneous catalytic reaction a reaction system, which consists of at least two independent stoichiometric equations. Typical multiroute catalytic processes are all oil processing (cracking, reforming, hydrotreating, and others), oxidation, dehydrogenation, and isomerization processes.

\subsection{Equilibrium in complex chemical reaction}

If we have $M$ reactions between $N$ chemical species (molecules) $A_{i}$, we can write

$$
\sum_{i=1}^{N} m_{j, i} \cdot A_{i}=0 j=1,2,3, \ldots . ., M ; i=1,2,3, \ldots . N
$$

where $m_{j, i}$ is the stoichiometric coefficients of $i^{\text {th }}$ specie in $j^{\text {th }}$ reaction.

The equilibrium in a complex chemical system can be described by using two matrixes. The atomic matrix $\mathrm{A}$ is built in such a way that the matrix rows correspond to the species present in the reaction mixture and the columns correspond to the atomic species, from which the reagents are composed. Each element $\left\|a_{i, j}\right\|$ of atomic matrix $A$ gives the number of atoms of certain sort, which contains certain reagent.

The molecular matrix $M$ is built in such a way that matrix rows correspond to the species present in the reaction mixture and the columns correspond to the possible reactions, in 
which these species can participate. The matrix element $\left\|m_{i, j}\right\|$ of molecular matrix $M$ is equal to the stoichiometric coefficient of the $i^{\text {th }}$ molecule in the $j^{\text {th }}$ reaction.

The relation between atomic $A$ and molecular matrix $M$ is given by the equation

$$
\|M\|^{T} \cdot A=0
$$

where $\|M\|^{T}$ is transposed molecular matrix. The maximal number of stoichiometrically independent chemical reactions, $M_{\mathrm{T}}$, in a complex reaction system is given by

$$
M_{\mathrm{T}}=N-q
$$

where $N$ is the total number of reagents in the system, and $q$ is the rank of the atomic matrix of the reagents.

The maximal number of stoichiometrically independent chemical reactions $M_{\mathrm{T}}$ forms the so-called stoichiometric (thermodynamic) basis for the reaction system, which is determined by the thermodynamics, the stoichiometry of the processes, and by the number and composition of the reagents. All chemical reactions in the system can be expressed as a linear combination of basic $M_{\mathrm{T}}$ reactions.

For every independent reaction, a single reagent named key component for this reaction exists. By measuring the concentrations of $M_{\mathrm{T}}$ key reagents, we are able to calculate the concentrations of the all reagents present in the reaction mixture.

\subsection{Reacting species}

The species participating in a heterogeneous catalytic reaction can be divided into two groups: reagents and intermediate surface compounds (ISC).

The reagents (non-Bodenstein reagents) are the initial compounds and products of the reactions. Their composition and structure are well known and their concentrations or partial pressures can be determined at any moment of the reaction and at any point in the reaction space.

The ISC (Bodenstein reagents) are formed as a result of the chemisorption of reagents on the catalyst surface. Most of them have a very short lifetime. For each chemisorbed reagent at least one ISC should be formed. The stoichiometry and structure of the ISC are not exactly known. In most of the cases, the concentrations of ISC cannot be determined directly and they are expressed by concentrations or partial pressures of the reagents via adsorption isotherms.

\subsection{Mass action and surface action law}

The presence of catalyst does not change the thermodynamic equilibrium but change only the reaction rates, via which the equilibrium is established. At equilibrium, the rates of forward and reverse reactions are equal (they can be very large or very small),

$$
r^{+}=r^{-}
$$

and overall reaction rate of the step is equal to zero,

$$
r=r^{+}-r^{-}=0
$$

The mass action law describes the relation between concentrations of reagents at equilibrium condition [12]. For reaction

$$
\alpha \mathrm{A}+\beta \mathrm{B}=\gamma \mathrm{C}+\delta \mathrm{D}
$$

the mass action law has the form

$\frac{P_{\mathrm{C}}^{\gamma} P_{\mathrm{D}}^{\delta}}{P_{\mathrm{A}}^{\alpha} P_{\mathrm{B}}^{\beta}}=K \quad\left(K\right.$ is the equilibrium constant $\left.K=\frac{k^{-}}{k^{+}}\right)$

where $P_{i}$ are the equilibrium partial pressures of the reagents.

Far from equilibrium, the mass action law has the form

$$
r^{+}-r^{-}=k^{+} P_{\mathrm{A}}^{\alpha} P_{\mathrm{B}}^{\beta}-k^{-} P_{\mathrm{C}}^{\gamma} P_{\mathrm{D}}^{\delta}
$$

where $r^{+}$and $r^{-}$are reaction rates at forward and back directions, $k^{+}$and $k^{-}$are corresponding reaction rate constants, and $\alpha, \beta, \gamma$, and $\delta$ are the reaction rate orders.

Since the heterogeneous catalytic reaction takes place on catalyst surfaces the mass action law is transformed to surface action law valid for processes taking place on catalyst surface $[13,14]$. In this case, the Eq. (6) became

$$
\alpha \mathrm{A}+\beta \mathrm{B}+\mu_{1} \theta_{1}+\mu_{2} \theta_{2}=\gamma \mathrm{C}+\delta \mathrm{D}+\mu_{3} \theta_{3}+\mu_{4} \theta_{4}
$$

and Eq. (8) will be transformed to

$$
r^{+}-r^{-}=k^{+} P_{\mathrm{A}}^{\alpha} P_{\mathrm{B}}^{\beta} \theta_{1}^{\mu_{1}} \theta_{2}^{\mu_{2}} \theta_{0}^{\Delta \mu}-k^{-} P_{\mathrm{C}}^{\gamma} P_{\mathrm{D}}^{\delta} \theta_{3}^{\mu_{3}} \theta_{4}^{\mu_{4}}
$$

where $P_{\mathrm{A}}, P_{\mathrm{B}}, P_{\mathrm{C}}$, and $P_{\mathrm{D}}$ are partial pressures of the reagents that participate in the reaction from the gas phase, $\theta_{0}$ is the surface concentration of free active sites, $\theta_{1}, \theta_{2}, \theta_{3}$, and $\theta_{4}$ are surface concentrations of the ISC, which participate in the reaction and $\Delta \mu=-\mu_{1}-\mu_{2}+\mu_{3}+\mu_{4}$ is the sum showing whether reaction is proceeding with the change of number of particles or not. If $\Delta \mu>0$, reaction proceeds with increasing the number of particles; if $\Delta \mu<0$, reaction proceeds with decreasing the number of particles and multiplier $\theta_{0}^{\Delta \mu}$ appears in the second part of Eq. (10).

\subsection{Ideal adsorption layer}

Ideal adsorption layer is an analogue of ideal gases valid for surface phenomena. It is defined by the following characteristics:

- The chemisorption process is described by Langmuir isotherm;

- All active centers on the surface are energetically equal;

- On every active center only one molecule or atom is adsorbed;

- Adsorbed species does not interact with each other.

\subsection{Reaction rate}

The absolute measure for catalytic activity is the reaction 
rate. Reaction rate is expressed by the number of moles reacted per unit time in unit reaction space at fixed composition of the reaction mixture and fixed degree of conversion. In case of the heterogeneous catalytic reactions, the catalyst surface can be regarded as reaction space. Therefore the reaction rate of heterogeneous catalytic reaction can be expressed by the number of moles reacted per unit time on unit catalyst surface area. The catalyst surface area can be replaced by some related quantities like catalyst weight, catalyst volume, number of active centers per unit area etc. For a closed system we can write

$$
r_{i}=-\frac{\mathrm{d} N}{\Omega \cdot \mathrm{d} t}=-\frac{V \cdot \mathrm{d} C_{i}}{\Omega \cdot \mathrm{d} t}=-\frac{V \cdot P_{i} \cdot \mathrm{d} x}{\Omega \cdot R T \cdot \mathrm{d} t}
$$

where $r$ is the reaction rate, $N_{i}$ is the number of moles of reactant $i, C_{i}$ is mole concentration of the reactant $i, V$ is the volume of the closed system, $\Omega$ is the reaction space (catalyst surface area, catalyst weight, catalyst volume, number of active centers per unit area), $P$ is partial pressure of reactant $i$, and $x$ is the degree of conversion.

For an open system we have

$$
r=-\frac{\mathrm{d} x}{\mathrm{~d}\left(\frac{W}{F}\right)}=-\frac{F \cdot \mathrm{d} x}{W}=-\frac{\mathrm{d} C}{\mathrm{~d}\left(\frac{W}{F}\right)}=-\frac{F \cdot \mathrm{d} C}{W}
$$

where $W / F$ is the contact time [volume/(volume/time) $=$ time], $F$ is the flow rate in moles per time, $W$ is the amount or volume of the catalysts charged into reactor, $x$ is degree of conversion, and $C$ is the concentration of reactant. One should have in mind that concentration in a flow system is determined from ratio of flow rate $F$ and volumetric flow rate $v$, i.e. $C=F / v=($ moles $/$ time $) /($ litters $/$ time $)=$ moles $/$ litter.

For complex catalytic reactions, measure of catalytic activity should be reaction rate on independent stoichiometric routes as defined below. The rate of the reaction on stoichiometric independent routes (which we cannot measure experimentally) is calculated from the reaction rates for the individual reagents in the system (which can be measured directly during the experiment) using the equation

$$
r_{i}=\sum_{j=1}^{M_{\mathrm{T}}}\left\|\mu_{i, j}\right\|^{T} R_{j}
$$

where $r_{\mathrm{i}}$ is the reaction rate for $i^{\text {th }}$ reagent, $i=1,2, \ldots, n$ is the number of the reagents in the system, $R_{j}$ is the reaction rate along the stoichiometric linearly independent route $j$, and $j=1,2, \ldots . ., M_{\mathrm{T}},\left\|\mu_{i, j}\right\|^{T}$ is an element of transposed stoichiometric (molecular) matrix of stoichiometrically independent routes. Each element $\left\|\mu_{i, j}\right\|^{T}$ of the stoichiometric matrix gives the stoichiometric coefficient of $i^{\text {th }}$ reagent in $j^{\text {th }}$ independent reaction route.

This means that for complex reaction instead of one reaction rate we will use a vector, which elements are reaction rates on each independent reaction route. In multiroute reac- tion system, we have to calculate the selectivities on stoichiometric independent routes, which are expressed as a ratio of reaction rate on the selected route to the sum of reaction rates on all independent routes.

$$
S_{j}=\frac{R_{j}}{\sum_{j=1}^{j=M_{\mathrm{T}}} R_{j}}
$$

The so-called turnover number [15] is another definition of the reaction rate. The turnover number is equal to the number of molecules reacted on one active surface center per unit time. The number of active surface centers in industrial catalysts however, cannot be estimated very precisely, which leads to the big errors in the estimated reaction rate. Therefore, it is recommendable not to use the turnover number for characterization of the activity of industrial catalysts.

\subsection{Rate constant and activation energy}

The relation between rate constant and activation energy $E$ as a function of the temperature is expressed by the Arrhenius equation

$$
k=k_{0} \exp \left(\frac{-E}{R T}\right)
$$

where $k^{0}$ is a preexponential factor.

For reaction of first order, the rate constant is equal to the reaction rate at concentration of reactant equal to one.

The Arrhenius equation is strictly valid only for processes, in which one energy barrier is overcome. That means that it is strictly valid only for elementary reactions.

All catalytic reactions are multistep processes, and therefore for them the Arrhenius equation is not strictly valid but it is used as convenient approximation.

\subsection{Principle of microscopic reversibility}

At equilibrium any microscopic process is reversible, which means that any step of a given process should be self-balanced [16]. That means that:

- The reaction should proceed in both directions via the same intermediate compounds;

- Adsorption-desorption processes in both directions should be the same;

- Active surface centers should be the same for the processes in both directions;

- If reaction is proceeding through a consequence set of elementary steps in forward direction the reverse process has to pass through the same set in reverse direction;

- If the process $A \rightarrow B$ is proceeding via the set of elementary steps $\mathrm{A} \rightarrow \mathrm{A}_{1} \rightarrow \mathrm{A}_{2} \rightarrow \mathrm{A}_{3} \rightarrow \mathrm{B}$, the reverse proc- 
ess $\mathrm{B} \rightarrow \mathrm{A}$ should proceed in following way $\mathrm{B} \rightarrow \mathrm{A}_{3} \rightarrow \mathrm{A}_{2} \rightarrow \mathrm{A}_{1} \rightarrow \mathrm{A}$;

- If reaction mechanism has a rate-limiting step it should be the same in both directions.

\subsection{Elementary steps}

Every heterogeneous catalytic reaction proceeds via a certain number of elementary reactions. Elementary reaction is the reaction, in which only one energy barrier is overcome. The stoichiometric coefficients of elementary reactions are integers. The rate of elementary reaction obeys the Law of Mass Action, or the Law of Surface Action, if the reactions take place at the surface of a catalyst.

Two elementary reactions proceeding in forward and reverse directions are forming the elementary step. Three types of elementary steps are defined: mass-transfer steps, adsorption-desorption steps, and surface reaction steps. If the elementary reaction is irreversible, the elementary step consists of one elementary reaction. In elementary reactions, participants are the ISC or the ISC together with reagents.

The rate of the $s^{\text {th }}$ elementary step connecting the $\alpha$ and $\beta$ ISC

$$
\sum_{j=1}^{m} \mu_{j, s} P_{j}+X_{\alpha}=\sum_{i=1}^{m^{\prime}} \mu_{i, s} P_{i}+X_{\beta}
$$

is therefore being expressed as:

$$
r_{s}=r_{s}^{+}-r_{s}^{-}=k_{s}^{+} X_{\alpha} \prod_{j=1}^{m} P_{j}^{\mu_{j s}}-k_{s}^{-} X_{\beta} \prod_{i=1}^{m^{\prime}} P_{i}^{\mu_{i s}}
$$

where $r_{\mathrm{s}}$ is the rate of the $s^{\text {th }}$ elementary reaction, $X_{\alpha}$ and $X_{\beta}$ are the concentrations of ISC $\alpha$ and $\beta$ involved in the $s^{\text {th }}$ step, $P_{j}$ and $P_{i}$ are the concentrations of the $j^{\text {th }}$ and $i^{\text {th }}$ reagents participating in the $s^{\text {th }}$ elementary step, $\mu_{j, s}$ and $\mu_{i, s}$ are the stoichiometric coefficients of the $j^{\text {th }}$ and $i^{\text {th }}$ reagents participating in the $s^{\text {th }}$ elementary step, and $m$ and $m^{\prime}$ are the number of reagents taking part in the forward and reverse elementary reactions of the $s^{\text {th }}$ step.

Linear step is a step, in which in both sides of the balance equation participate only one ISC. Equation (16) is an example of linear elementary step.

Existing experimental data show that in most of the cases in elementary reactions $\mu_{j, s}$ and $\mu_{i, s}=0$ or 1 , and $m$ and $m^{\prime}=$ 0 or 1 . In this case, Eq. (16) can be expressed as:

$$
P_{j}+X_{\alpha}=P_{i}+X_{\beta} \text { or } X_{\alpha}=X_{\beta}
$$

and Eq. (14) will take the form:

$$
r_{s}=k_{s}^{+} X_{\alpha} P_{j}-k_{s}^{-} X_{\beta} P_{i} \text { or } r=k_{s}^{+} X_{\alpha}-k_{s}^{-} X_{\beta}
$$

depending on whether the reagents participate in the $s^{\text {th }}$ elementary reaction or not.

If the $s^{\text {th }}$ elementary step is irreversible then Eq. (19) becomes

$$
r_{s}=k_{s}^{+} X_{\alpha} P_{j} \text { or } r_{s}=k_{s}^{+} X_{\alpha}
$$

\subsection{Linear relationships in the kinetics of heterogeneous catalytic reactions}

Polanyi equation $[17,18]$ states that for family of exothermic elementary reactions the linear relationship exists, which correlates the known thermodynamic properties and kinetic parameters of the steps

$$
E=E_{0}+\alpha \cdot \Delta H
$$

where $E$ is the observed energy of activation of the reaction, $\Delta H$ is the enthalpy of the reaction, $E_{0}$ is a constant for a given family of reactions, and $\alpha$ has value between 0 and 1 , usually $\alpha=0.5$.

For acid-based catalyzed reactions in liquid phase, Brönsted $[19,20]$ proposed the equation which relates reaction rate constant $k_{\mathrm{A}}$ and ionic strength of the acid represented by its dissociation constant $K_{\mathrm{A}}$,

$$
k_{\mathrm{A}}=C \cdot K_{\mathrm{A}}^{\alpha}
$$

where $C$ is constant for a given reaction and $\alpha$ is a transfer coefficient between 0 and 1 .

Temkin [21] extended the application of Eqs. (21) and (22) to heterogeneous catalysis phenomena. The equation below describes the relation between rate constants $k_{s}$ and the equilibrium constant $K_{s}$ of the elementary step "s":

$$
k_{s}=g \cdot K_{s}^{\alpha}
$$

where $g$ is parameter and $\alpha$ has value between 0 and 1 , usually $\alpha=0.5$. The Temkin equation is based on the acceptance that linear relation exists between kinetic and thermodynamic characteristics of the elementary steps in the adsorption and catalytic processes i.e.

$$
E_{s}=Q_{0}+\alpha \cdot Q_{S}
$$

where $E_{s}$ is energy of activation of the elementary step " $s$ ", $Q_{s}$ is heat effect of the elementary step " $s$ ", $Q_{0}$ is parameter, and $\alpha$ is between 0 and 1, usually $\alpha=0.5$.

The validity of Eqs. (23) and (24) means that these relations do not change in transition from one part of nonuniform catalyst surface to another part of the surface.

\subsection{Reaction mechanism}

The mechanism of chemical reaction is a set of elementary reactions that allow a qualitative and quantitative description of major characteristics of the studied process.

A mechanism consisting only of linear steps will be described as a linear mechanism. A large number of reactions proceed via non-linear mechanism, e.g. they include elementary reactions, having rates that depend non-linearly on the concentration of the ISC. The mechanism of process of selective (I) and total (II) oxidation of ethylene on a silver 
catalyst given below (25) is an example of mechanism containing linear (1) and (2) and non-linear (3) and (4) steps [19].

\begin{tabular}{lcc}
\hline \multirow{2}{*}{ Reaction mechanism steps } & \multicolumn{2}{c}{ Stoichiometric number } \\
\cline { 2 - 3 } & Route I & Route II \\
\hline (1) $\quad \mathrm{Z}+\mathrm{O}_{2}=\mathrm{ZO}_{2}$ & 2 & 0 \\
(2) $\quad \mathrm{ZO}_{2}+\mathrm{C}_{2} \mathrm{H}_{4} \rightarrow \mathrm{ZO}+\mathrm{C}_{2} \mathrm{H}_{4} \mathrm{O}$ & 2 & 0 \\
(3) $\quad 2 \mathrm{ZO}=2 \mathrm{Z}+\mathrm{O}_{2}$ & 1 & -3 \\
(4) $\quad \mathrm{C}_{2} \mathrm{H}_{4}+6 \mathrm{ZO} \rightarrow 2 \mathrm{CO}_{2}+\mathrm{H}_{2} \mathrm{O}+6 \mathrm{Z}$ & 0 & 1 \\
\hline
\end{tabular}

Route I: $2 \mathrm{C}_{2} \mathrm{H}_{4}+\mathrm{O}_{2}=2 \mathrm{C}_{2} \mathrm{H}_{4} \mathrm{O}$

Route II: $\mathrm{C}_{2} \mathrm{H}_{4}+3 \mathrm{O}_{2}=2 \mathrm{CO}_{2}+2 \mathrm{H}_{2} \mathrm{O}$

For the mechanism schemes presented, the stoichiometric numbers are given at the right side of every elementary step. They might be different in different reaction routes. The stoichiometric number equal to zero means that this particular elementary step does not take part in the given reaction route. Negative sign of the stoichiometric number means that this elementary step should be read from right to left. Mechanism of ethylene oxidation is an example for two route mechanisms. Every route has its own set of stoichiometric numbers. The first route consists of steps (1), (2), and (3) with stoichiometric numbers $\sigma_{1}^{(1)}=2, \sigma_{2}^{(1)}=2$, and $\sigma_{3}^{(1)}=1$. The second route consists of steps (3) and (4) with stoichiometric numbers $\sigma_{3}^{(2)}=-3$ and $\sigma_{4}^{(2)}=1$. The third elementary step participates in both routes with different stoichiometric numbers. The sign minus of $\sigma_{3}^{(2)}$ means that elementary step (3) in the second route is proceeding in the reverse direction.

\subsection{Reaction routes}

Complex reactions can be expressed as a manifold of elementary steps and ISC combination, which in certain conditions give different reaction pathways with producing the same or different products. Such pathways are named reaction routes [22-26]. Summing stoichiometric equations of elementary steps in given route multiplied by their stoichiometric numbers $\sigma$ one should obtain stoichiometric equation of the route. The stoichiometric number shows how many times a given elementary reaction should be repeated in order to have one turnover of the main reaction. Reaction rate on the reaction route is equal to the number of realization of reaction route in unit of time.

For any independent route, there is an elementary step $s$ called basic, which does not participate in any other reaction route. Its reaction rate $r_{s}$ will be given by the difference in the rates of elementary reactions in the forward $r_{s}^{+}$and reverse $r_{s}^{-}$directions:

$$
r_{s}=r_{s}^{+}-r_{s}^{-}=\sigma_{s} R
$$

where $\sigma_{\mathrm{s}}$ is the stoichiometric number of the elementary step $s$ in the reaction mechanism, and $R$ is the reaction rate along the route.

If the elementary step participates in $p$ routes, the rate of the elementary step $r_{s}$ will equal the sum of the rates along the different routes, in which the elementary step participates, multiplied by the stoichiometric number $\sigma_{s}^{(\mathrm{P})}$ of the elementary step for the given route:

$$
r_{s}=r_{s}^{+}-r_{s}^{-}=\sum_{P=1}^{\mathrm{P}} \sigma^{(P)} R^{(P)}
$$

where $\sigma_{s}^{(P)}$ is the stoichiometric number of the elementary step $s$ for the $P^{\text {th }}$ route, $R^{(\mathrm{P})}$ is the reaction rate along the $P^{\text {th }}$ route, and $P$ is the number of routes, in which participates the elementary step $s$.

The rates of the reaction on stoichiometrically independent routes (which we cannot measure experimentally) are calculated from the reaction rates for the individual reagents in the system (which can be measured directly during the experiment) using the Eq. (13).

The rate of each independent reaction route " $q$ " will be equal to the rate of its basic reaction step " $q$ " divided by the stoichiometric number of this reaction step in " $q$ " route

$$
R=\frac{r^{(q)}}{\sigma^{(q)}}=\frac{r_{s}^{+(q)}-r_{s}^{-(q)}}{\sigma^{(q)}}
$$

where the reaction rate for the step " $q$ ", $r^{(\mathrm{q})}$, is obtained from Eq. (29).

$$
r^{(q)}=r_{s}^{+(q)}-r_{s}^{-(q)}=k_{s}^{+(q)} X_{\alpha} \prod_{j=1}^{m} P_{j}^{\mu_{j s}}-k_{s}^{-(q)} X_{\beta} \prod_{i=1}^{m^{\prime}} P_{i}^{\mu_{i s}}
$$

Thus, the Eq. (29) states in explicit form the rates along the independent routes as a function of the concentrations of the reagents and the ISC participating in the basic steps, which makes it very convenient for solving practical problems.

\subsection{Horiuti's rule}

Horiuti's rule that is valid for both linear and non-linear mechanisms states that according to the complexity and structure of the proposed reaction mechanism (which depends upon our knowledge about the studied process, properties and number of ISC participating in the mechanism), the number of independent routes $M_{\mathrm{K}}$ in a complex reaction system is given by the following expression:

$$
M_{\mathrm{K}}=S-L_{\text {total }}+1
$$

where $S$ is the number of elementary steps in the reaction mechanism, and $L_{\text {total }}$ is the total number of ISC taking part in the reaction mechanism.

The reaction basis given by Eq. (30) is named kinetic basis. It consists of the routes obtained from proposed reaction mechanism. Their number is different for different mechanisms and depends on the details of the mechanism. It is 
obvious that $M_{\mathrm{K}} \geq M_{\mathrm{T}}$, which means that some of the routes of the kinetic basis are dependent from the thermodynamic point of view.

We now illustrate what has been said so far by the presenting of the mechanism of the reactions of isomerization of 1-butene, cis-2-butene and trans-2-butene as an example.

\begin{tabular}{llcccc}
\hline \multirow{2}{*}{ Reaction mechanism steps } & \multicolumn{5}{c}{ Route } \\
\cline { 3 - 6 } & I & II & III & IV \\
\hline (1) & $1-\mathrm{C}_{4} \mathrm{H}_{8}+\mathrm{Z}=1-\mathrm{C}_{4} \mathrm{H}_{8} \mathrm{Z}$ & 1 & 1 & 0 & 0 \\
(2) & cis $-2-\mathrm{C}_{4} \mathrm{H}_{8}+\mathrm{Z}=$ cis-2- $\mathrm{C}_{4} \mathrm{H}_{8} \mathrm{Z}$ & -1 & 0 & 1 & 0 \\
(3) & trans $-2-\mathrm{C}_{4} \mathrm{H}_{8}+\mathrm{Z}=$ trans $-2-\mathrm{C}_{4} \mathrm{H}_{8} \mathrm{Z}$ & 0 & -1 & -1 & 0 \\
(4) & $1-\mathrm{C}_{4} \mathrm{H}_{8} \mathrm{Z}=$ cis $-2-\mathrm{C}_{4} \mathrm{H}_{8} \mathrm{Z}$ & 1 & 0 & 0 & 1 \\
(5) & $1-\mathrm{C}_{4} \mathrm{H}_{8} \mathrm{Z}=$ trans $-2-\mathrm{C}_{4} \mathrm{H}_{8} \mathrm{Z}$ & 0 & 1 & 0 & -1 \\
(6) & cis $-2-\mathrm{C}_{4} \mathrm{H}_{8} \mathrm{Z}=$ trans $-2-\mathrm{C}_{4} \mathrm{H}_{8} \mathrm{Z}$ & 0 & 0 & 1 & 1 \\
\hline
\end{tabular}

I: $1-\mathrm{C}_{4} \mathrm{H}_{8}=$ cis $-2-\mathrm{C}_{4} \mathrm{H}_{8}$

II: $1-\mathrm{C}_{4} \mathrm{H}_{8}=$ trans $-2-\mathrm{C}_{4} \mathrm{H}_{8}$

III: cis-2- $\mathrm{C}_{4} \mathrm{H}_{8}=$ trans $-2-\mathrm{C}_{4} \mathrm{H}_{8}$

IV: $0=0$

Here $\mathrm{Z}$ is a free site on the catalyst surface, and $1-\mathrm{C}_{4} \mathrm{H}_{8} \mathrm{Z}$, cis- $\mathrm{C}_{4} \mathrm{H}_{8} \mathrm{Z}$, and trans $-\mathrm{C}_{4} \mathrm{H}_{8} \mathrm{Z}$ are ISC for three butenes.

For this particular mechanism, the stoichiometric basis $M_{\mathrm{T}}$ and the kinetic basis $M_{\mathrm{K}}$ are given by following equation:

$M_{\mathrm{T}}=N-q=3-1=2$ and $M_{\mathrm{K}}=S-L_{\text {total }}-1=6-4+1=3$

We have two stoichiometrically independent routes in the thermodynamic basis and three independent routes in the kinetic basis of this mechanism. It follows that one route of kinetic basis is stoichiometrically dependent. If we find that the third route is stoichiometrically dependent, two opportunities exist for making $M_{\mathrm{T}}$ equal to $M_{\mathrm{K}}$ :

(i) To remove from the mechanism one elementary step so $M_{\mathrm{K}}=5-4+1=2$;

(ii) To preserve the proposed mechanism by changing the stoichiometric numbers of the elementary steps participating in the third route and it will turn to null route. Such opportunity is presented as route IV. The reaction along this route proceeds at a finite rate without influencing the stoichiometry of the process.

The relationship between the reaction rates for the reagents and the rates along the linearly independent stoichiometric routes are expressed by the following system:

$$
\begin{gathered}
r_{1 \text {-butene }}=R(\mathrm{I})-R(\mathrm{II}) \\
r_{\text {cis-2-butene }}=R(\mathrm{I}) \\
r_{\text {trans-2-butene }}=R(\mathrm{II})
\end{gathered}
$$

\subsection{Steady state approximation}

The steady state regime of the proceeding of a given chemical process can be realized only in open systems $[27,28]$. The steady state regime means that all reaction parameters (concentrations of reagents and ISC, temperatures, partial and total pressure, regents flow rate) should have constant values, which do not change with time. This however does not mean that the parameters should have the same value at different points of the reaction space. For the changes of the surface concentration $X_{i}$ of every ISC (Bodenstein reagents) we can write

$$
\frac{\mathrm{d} X_{i}}{\mathrm{~d} t}=F\left(c_{j}, X_{\mathrm{k}}\right)
$$

where function $F\left(c_{j}, X_{\mathrm{k}}\right)$ is a balance of reacting species that allow us to express the concentrations of each ISC via the concentrations of the reagents, $j=1,2, \ldots \ldots \ldots, N$ is the number of reagents in the system, and $i=1,2, \ldots ., K$ is the number of ISC (including free catalyst surface) formed on the catalyst surface and included in the reaction mechanism $K \geq N+1$.

At steady state regime Eq. (33) becomes

$$
\frac{\mathrm{d} X_{i}}{\mathrm{~d} t}=0
$$

That means that the realization of the steady state regime leads to very important simplification in the treatment of kinetic problems. In order to find all concentrations of ISC instead of solving the system (one equation for every ISC) of differential Eq. (33), we have to solve the system containing the same number of algebraic Eq. (35) and substantially to simplify the problem.

$$
\sum_{i=1}^{K} F\left(c_{j}, X_{\mathrm{k}}\right)=0
$$

The steady state regime means that:

(i) The rate of formation of the $i^{\text {th }}$ ISC from all possible reactions should be smaller than the rate of its consumption along all possible reactions

$$
\frac{1}{X_{i}} \frac{\mathrm{d} X_{i}}{\mathrm{~d} t} \ll \frac{1}{X_{i}}\left|\sum_{i} r_{i}\right|
$$

(ii) The life-time $\tau_{i}^{*}$ of the $i^{\text {th }}$ ISC should be nearly equal to the time necessary to reach steady state $\tau_{\mathrm{o}}$ and smaller than the time of reaction $\tau_{\mathrm{r}}$,

$$
\tau_{i}^{*} \approx \tau_{\mathrm{o}}<\tau_{\mathrm{r}}
$$

(iii) The reaction rates of all consecutive elementary steps should be approximately equal to each other and equal to the slowest one called limiting reaction step $s_{\mathrm{L}}$. All other steps are faster and we can accept that they are in equilibrium. For each step we can write

$$
\begin{array}{r}
r_{1}^{+}-r_{1}^{-}=\sigma_{1} r, \quad r_{2}^{+}-r_{2}^{-}=\sigma_{2} r, \ldots \ldots \ldots \\
r_{s}^{+}-r_{s}^{-}=\sigma_{s} r, \quad r_{L}^{+}-r_{L}^{-}=\sigma_{L} r
\end{array}
$$

or

$$
r=\frac{r_{1}^{+}-r_{1}^{-}}{\sigma_{1}}=\frac{r_{2}^{+}-r_{2}^{-}}{\sigma_{2}}=\ldots \ldots \ldots . . .=\frac{r_{s}^{+}-r_{s}^{-}}{\sigma_{s}}=\frac{r_{L}^{+}-r_{L}^{-}}{\sigma_{L}}
$$

where $r_{s}^{+}$and $r_{s}^{-}$are the reaction rates in the straight and reverse directions of the $s^{\text {th }}$ elementary step and $\sigma_{\mathrm{s}}$ is the 
stoichiometric number of the $s^{\text {th }}$ elementary steps in given reaction mechanism.

The stoichiometric numbers vary usually between 1 and 3 . Therefore, the difference between the rates of the elementary steps in a given reaction route can reach one order of magnitude. This difference depends upon the numbers of reaction routes in which this step is participating and values of the stoichiometric numbers in every one of them.

As a conclusion, we can say that at steady state approximation we deal with the kinetics of the limiting reaction steps and with thermodynamics of the fast elementary reaction step since they are always in equilibrium.

At nonsteady state regime, it is possible that the rate-limiting step will be different from rate-limiting step at steady state conditions.

\subsection{Application of the steady state approximation for deriving the reaction rate equations}

Solving Eq. (35) for the reaction mechanism, which consists of $s$ elementary steps, we obtain the following equation:

$$
r=\frac{r_{1}^{+} r_{2}^{+} \ldots r_{s}^{+}-r_{1}^{-} r_{2}^{-} \ldots r_{s}^{-}}{\sigma_{1} r_{2}^{+} \ldots r_{s}^{+}+r_{1}^{-} \sigma_{2} r_{3}^{+} \ldots r_{s}^{+}+\ldots+r_{1}^{-} r_{2}^{-} \ldots r_{s-1}^{-} \sigma_{s}}
$$

in which the overall rate is expressed by the rates of the elementary reactions participating in the reaction mechanism. Equation (40) can be rearranged in form, which is very convenient for practical applications.

$$
r\left(\frac{\sigma_{1}}{r_{1}^{+}}+\frac{r_{1}^{-} \sigma_{2}}{r_{1}^{+} r_{2}^{+}}+\ldots . . \frac{r_{1}^{-} r_{2}^{-} \ldots . . r_{s-1}^{-} \sigma_{s}}{r_{1}^{+} r_{2}^{+} \ldots . . r_{s}^{+}}\right)=1-\frac{r_{1}^{-} r_{2}^{-} \ldots . . r_{s}^{-}}{r_{1}^{+} r_{2}^{+} \ldots . . r_{s}^{+}}
$$

If the reacting system is described by $P$ reaction routes, the Eq. (41) will be extended to the following system of rate equations:

$$
\sum_{p=1}^{P} r^{(P)}\left(\frac{\sigma_{1}^{(P)}}{r_{1}^{+}}+\frac{r_{1}^{-} \sigma_{2}^{(P)}}{r_{1}^{+} r_{2}^{+}}+\ldots . . \frac{r_{1}^{-} r_{2}^{-} \ldots . . r_{s-1}^{-} \sigma_{s}^{(P)}}{r_{1}^{+} r_{2}^{+} \ldots . . r_{s}^{+}}\right)=1-\frac{r_{1}^{-} r_{2}^{-} \ldots . . r_{s}^{-}}{r_{1}^{+} r_{2}^{+} \ldots . . r_{s}^{+}}
$$

This is the general rate equations system derived by Temkin, which can be used for deriving kinetic models without limitation due to characteristics of the reaction mechanism.

Every term of the left side of the Eqs. (41) and (42) corresponds to a given ISC. Except for the first term, in the other terms the nominator contains the rates for the reversed direction for the previous steps and denominator contains all the rates for the following steps for forward directions. It means that the ISC produced in the steps in the nominator are consumed by the steps in the denominator and these concentrations disappear from the equation except the ISC produced in the first step. In order to obtain equations for all ISC in the system we have to change consecutively the number of the steps in such way that every step should appear once as a number one.

Let have a reaction with one route mechanism.

$$
\begin{array}{ll}
\text { (1) } A+Z=Z_{1} & \sigma_{1}=1 \\
\text { (2) } Z_{1}+B=Z_{2} & \sigma_{2}=1 \\
\text { (3) } Z_{2}=C+Z & \sigma_{3}=1 \\
A+B=C &
\end{array}
$$

According to the Mass Action Law the reaction rates of elementary reactions in the mechanism (43) are:

$$
\begin{array}{ll}
r_{1}^{+}=k_{1}^{+} P_{\mathrm{A}} \mathrm{Z} & r_{1}^{-}=k_{1}^{-} \mathrm{Z}_{1} \\
r_{2}^{+}=k_{2}^{+} P_{\mathrm{B}} \mathrm{Z}_{1} & r_{2}^{-}=k_{2}^{-} \mathrm{Z}_{2} \\
r_{3}^{+}=k_{3}^{+} \mathrm{Z}_{2} & r_{3}^{-}=k_{3}^{-} P_{\mathrm{C}} \mathrm{Z}
\end{array}
$$

where $\mathrm{Z}$ is the empty part of the catalyst surface, and $\mathrm{Z}_{1}$ and $Z_{2}$ are the surface concentrations of the ISC participating in the reaction mechanism. The ISC are connected by a balance equation $\mathrm{Z}+\mathrm{Z}_{1}+\mathrm{Z}_{2}=1$.

Inserting these expressions into the balance equation and accepting for simplicity that the second step of the reaction mechanism is irreversible i.e. $r_{2}^{-}=0$, we find the expression for the reaction rate equation:

$$
r=\frac{k_{1}^{+} k_{2}^{+} k_{3}^{+} P_{\mathrm{A}} P_{\mathrm{B}}}{k_{1}^{+} k_{3}^{+} P_{\mathrm{A}}+k_{2}^{+} k_{3}^{+} P_{\mathrm{B}}+k_{1}^{+} k_{2}^{+} P_{\mathrm{A}} P_{\mathrm{B}}}
$$

For written in a traditional form,

$$
r=\frac{k_{1} P_{\mathrm{A}} P_{\mathrm{B}}}{k_{2} P_{\mathrm{A}}+k_{3} P_{\mathrm{B}}+k_{4} P_{\mathrm{A}} P_{\mathrm{B}}}
$$

All constants in the Eqs. (45) and (46) have a complex character and are expressed by the different functions of the constants of the elementary reactions participating in the reaction mechanism (44). For this reason, the Arrhenius equation can be used for description of the dependence of the overall reaction rate from the temperature only as an approximation but not as an exact law.

\section{Stages of the building of kinetic models}

Building of kinetic models is a difficult and time-consuming process that is passing through several steps.

\subsection{Formulation of possible reaction mechanisms}

The first step is the formulation of the set of possible reaction mechanisms. The proposed mechanisms should be based on all available knowledge about the studied process and the used catalyst. That means that mechanism formulation shouldn't be based only on results from kinetic experiments. For this aim should be used the information about reaction chemistry, thermodynamics, structure and number of possible ISC, types and number active sites on the surface of catalysts, chemical properties of the solid catalysts, etc. This information can be obtained from:

- Published data in the scientific literature; 
- Kinetic experiments at steady state conditions, in which variations of the reaction parameters must be as wide as possible;

- Kinetic experiments at nonsteady state conditions in order to evaluate the features of the elementary steps different from rate-limiting step at steady state conditions;

- Results from physical and chemical adsorption measurements;

- Application of physical methods for characterization of the used catalyst;

- Application of chemical methods for characterization of the catalyst.

\subsection{Deriving steady state kinetic models corresponding to the proposed reaction mechanisms}

The second step is deriving kinetic equations corresponding to every proposed reaction mechanism. The reaction rate equations can be derived using different methods. We shall just mention the most popular methods. They are well known and it is not necessary to describe them in details. It is worth mentioning however, that kinetic parameters of models obtained by using different methods will have different physical meanings and different dimensions.

(i) Method of Hougen-Watson [29] is widely used. It is based on presumption of validity of ideal adsorption layer and the application of steady state approximation. The kinetic parameters in the denominator of these models have physical meaning of adsorption constants. For every mechanism, one can select different rate-limiting steps and therefore the set of plausible models might be quite big.

(ii) Method of Temkin [30,31] is based on the presumption that there are deviations from the postulates of the ideal adsorption layer. The theory of this method is based on idea that energy of adsorption on active centers on the catalyst surface is evenly distributed.

(iii) Method of Temkin for stationary heterogeneous complex catalytic reactions $[32,33]$. This is the most general method based on steady state approximation including all elementary steps in the used reaction mechanism. It does not include requirements for the type of distribution of active centers by their energy of adsorption. The kinetic parameters of the derived equations are functions of the kinetic constants of all elementary steps in reaction mechanism.

In some cases for mechanisms containing only linear elementary steps other more specific methods like method of graph theory [34-37] can be used. The method of graph theory might be regarded as a particular case of the method of Temkin for stationary heterogeneous complex catalytic reactions.

\section{Sources of experimental errors in studies of kinetics of catalytic heterogeneous reactions}

The sources of experimental errors during the studies of kinetics of catalytic heterogeneous reactions are result of influence of many factors:

- Quality of raw materials for the catalyst preparation;

- Technology of the catalyst production;

- Inhomogenity of catalyst samples due to the process of the catalyst preparation;

- Quality of the reactants;

- Inhomogenity of catalyst samples due to the processes of the catalyst pretreatments and regeneration;

- Changes in the reaction parameters during the catalytic experiments;

- Quality and preciseness of the analytical methods and other equipment;

- Type and quality of the selected of laboratory reactors;

- Heat and mass transfer influence.

We will not go into details and discuss all of them. Rather we will concentrate on the last two issues.

\section{Laboratory reactors}

We shall discuss in general terms some advantages and disadvantages of different types of laboratory reactors for their use in kinetic studies. General information about laboratory heterogeneous catalytic reactors can be found in [38].

\subsection{Closed (batch) reactor}

Basic principle - integral reactor; Main requirement ideal mixing; Basic condition - quasi steady state; Typical gradients - concentration with time; Advantages - small amount of reactant, precision of measurements, obtaining full kinetic curve in one experiment; Disadvantages - difficulties in achieving quasi steady state, no direct measurement of the reaction rate.

Reaction rate $r$ can be estimated from the equation

$$
t=N_{0} \int_{0}^{x} \frac{\mathrm{d} x}{r \cdot W}
$$

\subsection{Flow reactor}

Basic principle - integral reactor; Main requirement ideal plug flow; Basic condition - steady state regime; Typical gradients - radial and longitudinal gradients of concentration and temperature, the catalyst bypassing, channeling near the reactor wall; Advantages - similarity to industrial units, simple equipment; Disadvantages - difficulties in achieving quasi steady state, no direct measurement of the 
reaction rate, many gradients.

Reaction rate $r$ can be estimated from the equation

$$
\frac{W}{F}=\int_{0}^{x} \frac{\mathrm{d} x}{r}
$$

\subsection{Differential reactor}

Basic principle - differential reactor; Main requirement ideal plug flow; Basic condition - steady state, low degree of conversion up to $10 \%$, high space velocity; Typical gradients - radial and longitudinal gradients of concentration and temperature; Advantages - opportunity to measure directly the reaction rate, similarity to industrial units, simple equipment; Disadvantages - difficulties in achieving quasi steady state, existence of some gradients, difficult to apply for multiroute catalytic processes.

Reaction rate $r$ is estimated from the equation:

$$
r=\frac{F \cdot \Delta x}{W}
$$

\subsection{Gradientless reactor}

The reactor with external circulation was introduced in 1950 by Temkin, Kiperman, and Lukyanova [39]. The reactors with internal circulations were described by Berty [40,41].

Basic principle - differential reactor; Main requirement ideal mixing; Basic condition - rate of mixing >> flow rate; Typical gradients - in some cases gradients due to internal, diffusion limitations.

Advantages - direct measurement of reaction rate at any degree of conversion, absence of gradients. By increasing the circulation rate, mass velocity can be increased without changing the flow rate $F$ and, consequently the contact time $W / F$ and the overall space velocity remain unchanged. The opposite is also possible, keeping the circulation rate and mass velocity unchanged even if flow rate $F$, and with it the overall space velocity and constant time, is changed. Gradientless reactor permits with small fresh feed and small quantity of catalyst to achieve mass velocities of commercial reactors. It is possible to separate the concentration and temperature terms and to measure the apparent energy of activation even when the concentration dependency is not known. If two experiments are made at two temperatures and the discharge concentrations are the same (at different feed rates) then the observed effect is attributed to temperature.

Disadvantages - complicated equipment, not suitable for reaction with fast deactivating catalyst. The greatest difficulty with gradientless reactors is that discharge concentrations should be the independent variables along with temperature, while feed rate and even feed compositions will be dependent variables. In practical terms, this means that feed rate and feed composition have to be changed until the desired exit values are reached. In this sense contact time, or space velocities, becomes a dependent variable.

Reaction rate $r$ in a gradientless reactor is estimated from Eq. (49).

\section{Mass and heat transfer in the kinetics of heterogeneous catalytic reactions}

Heterogeneous catalytic reactions are always connected with processes of mass and heat transfer [2,42-46]. If these processes are fast enough, they do not have any substantial influence on the proceeding of the catalytic processes. When, however, these processes are slow enough the situation becomes different. Under steady state conditions, the rates of all consecutive steps should be equal to one another and equal to the slowest one. Since the diffusion steps are the slowest one, the overall reaction rate will be equal to the rate of the diffusion. In this case, the overall reaction rate will not be connected with the reaction mechanism but will be determined by the rate of physical processes. It is clear that the processes of mass transfer can have a substantial effect on the proceeding of heterogeneous catalytic reactions. Therefore, it is obvious that the study of the influence of the processes of mass and heat transfer on the proceeding of heterogeneous catalytic reactions has a great theoretical and practical importance. The following different regimes for proceeding of heterogeneous catalytic reaction may be observed:

(i) Kinetic regime in which the intrinsic surface reactions are the slowest and determine the overall reaction rate;

(ii) External diffusion regime in which the processes of mass transfer of the reagents and product of the reaction to and from the external catalyst surface are the slowest and determine the overall reaction rate;

(iii) Internal diffusion regime in which the processes of mass transfer of the reagents and product of the reaction within the pores of catalyst particles are the slowest and determine the overall reaction rate;

(iv) Transition regime in which both the chemical and mass transfer processes have approximately equal rates and the overall reaction rate is determined by the proceeding of the intrinsic surface reactions and diffusion.

In cases that it is not possible to carry out the process in kinetic regimes, building kinetic models that account for mass transfer processes is an important task from the practical point of view.

\subsection{Fundamental laws of transport phenomena}

In a stagnant binary gas mixture, according to the Fick's 
first law, the molar flux $J\left[\mathrm{~mol} /\left(\mathrm{cm}^{2} \cdot \mathrm{s}\right)\right]$ is proportional to the concentration gradient in the direction $x$ of the diffusion:

$$
J_{1}=-D_{1,2} \frac{\mathrm{d} c_{1}}{\mathrm{~d} x}=-D_{1,2} \cdot c_{\text {tot }} \frac{\mathrm{d} Y_{1}}{\mathrm{~d} x} c_{1}
$$

where $c_{1}$ is the molar concentration of the component $1, c_{\text {tot }}$ is the total molar concentration of the mixture, $Y_{1}$ is the mole part of the component $1, D_{1,2}\left[\mathrm{~cm}^{2} / \mathrm{s}\right]$ is the diffusion coefficient for 1 diffusing in gas 2 , and $x$ is the direction of the mass flow due to diffusion.

The diffusion coefficient is a function of the molecular properties of the two gases and increases with increase in temperature or decrease in pressure. It varies little with the mole fractions of the two gases but it varies more with the changes in the total molar concentration of the gas mixture $c_{\text {tot }}$.

The heat flow $q\left[\mathrm{cal} /\left(\mathrm{cm}^{2} \cdot \mathrm{s}\right)\right]$ from high to low temperature regions according to the Fourier's law is determined by the following equation:

$$
q=-k_{\mathrm{t}} \frac{\mathrm{d} T}{\mathrm{~d} x}
$$

where $k_{\mathrm{t}}$ is the thermal conductivity of the fluid, $T$ is the absolute temperature, and $x$ is the direction of the heat flow. The kinetic molecular theory gives expressions for both $D_{12}$ and $k_{\mathrm{t}}$.

When a fluid is passing over the surface of a grain, it forms a boundary layer, in which the velocity parallel to the surface varies rapidly over a very short distance normal to the flow. At the solid surface, the fluid velocity is zero, but approaches the bulk-stream velocity in the distance less than millimeter away from the surface. In this thin layer the mass and heat transfer are proceeding via molecular diffusion, while in the main fluid stream mass transfer is essentially independent of the molecular diffusion coefficient. Therefore, the overall mass transfer is proportional to the molecular diffusion coefficient. The molar flow rate $N$ $\left[\mathrm{mol} /\left(\mathrm{cm}^{2} \cdot \mathrm{s}\right)\right]$ is given by

$$
N=k_{c}\left(C_{0}-C_{\mathrm{s}}\right)
$$

where $N$ is the diffusion flux fluid to solid, $k_{\mathrm{c}}$ is the coefficient of mass transfer, $C_{\mathrm{s}}$ is the concentration at the surface, and $C_{0}$ is the concentration in the ambient bulk fluid.

In gas system, partial pressures should be used instead of concentrations

$$
N=k_{\mathrm{G}}\left(p_{0}-p_{\mathrm{s}}\right)
$$

where $k_{\mathrm{G}}=k_{\mathrm{c}} / R T, k_{\mathrm{G}}$ and $k_{\mathrm{c}}$ are related to the molecular diffusion coefficient $\left(D_{1,2}\right)$ and the thickness of the boundary layer $(\delta)$ by the following relation:

$$
k_{\mathrm{c}}=\frac{D_{1,2}}{\delta \cdot k_{\mathrm{G}}}=\frac{D_{1,2}}{\delta \cdot R T}
$$

The heat flow through the boundary layer can be expressed as:

$$
q=h\left(T_{\mathrm{b}}-T_{\mathrm{s}}\right)
$$

where $h$ is the heat transfer coefficient related to the thermal conductivity of the fluid and the size of the boundary layer.

The data on mass and heat transfer between bulk stream and particle surface can be presented by dimensionless groups. For mass transfer phenomena, three dimensionless criteria can be defined:

(i) Reynolds number

$$
\operatorname{Re}=\frac{G \cdot d_{\mathrm{p}}}{\mu}
$$

(ii) Schmidt number

$$
S_{c}=\frac{\mu}{D \cdot \rho}
$$

(iii) Sherwood number

$$
S h=\frac{k_{\mathrm{c}} \cdot \rho}{G}
$$

where $G$ is the mass velocity based upon the cross-sectional area of empty reactor, $\mu$ is the viscosity of the fluid, $d_{\mathrm{p}}$ is the diameter of catalyst particles, $\rho$ is the density of fluid, and $D$ is molecular diffusivity of the transferred component.

The coefficient of mass transfer $k_{\mathrm{c}}$ can be estimated using the so-called $j$-factor defined by the following expression:

$$
j_{\mathrm{D}}=S h \cdot S c^{3 / 2}=\frac{\alpha}{\varepsilon} R e^{-\beta}
$$

and

$$
k_{\mathrm{c}}=\frac{\alpha \cdot R e^{-\beta} \cdot G \cdot D^{1.5} \cdot \rho^{0.5}}{\varepsilon \cdot M^{1.5}}=j_{\mathrm{D}} G \cdot D^{1.5} \rho^{0.5}
$$

where $\varepsilon$ is a void fraction of the catalyst bed, and $\alpha$ and $\beta$ are constants, which must be determined experimentally. For gases when $3<R e<2000$ and $0.416<\varepsilon<0.778, \alpha=$ 0.357 and $\beta=0.359$. For liquids when $55<R e<1500$ and $0.35<\varepsilon<0.75, \alpha=0.250$ and $\beta=0.310$.

The data on heat transfer are correlated in the same way as those on mass transfer. For heat transfer phenomena, the Prandtl number is defined as:

$$
\operatorname{Pr}=\frac{\mu \cdot C_{\mathrm{p}}}{k_{\mathrm{fld}}}
$$

where $C_{\mathrm{p}}$ is the specific heat of the fluid, $k_{\mathrm{fld}}$ is the thermal conductivity of the fluid, and $\mu$ is the viscosity of the fluid. Then for $j_{\mathrm{H}}$ and heat transfer coefficient $h$ we have the equations

$$
j_{\mathrm{H}}=\frac{h \cdot P r^{2 / 3}}{C_{\mathrm{p}} \cdot G}=\frac{\alpha}{\varepsilon} \operatorname{Re}^{-\beta}
$$

and

$$
h=\frac{\alpha \cdot R e^{-\beta} \cdot C_{\mathrm{p}}^{1 / 3} \cdot G \cdot k_{\mathrm{fld}}}{\varepsilon \mu^{2 / 3}}=\frac{j_{\mathrm{H}} \cdot C_{\mathrm{p}}^{2 / 3} \cdot k_{\mathrm{fld}}}{\mu^{2 / 3}}
$$

Between $j_{\mathrm{D}}$ and $j_{\mathrm{H}}$ factors, there exists a correlation

$$
j_{\mathrm{H}}=1.08 j_{\mathrm{D}}
$$

\subsection{External diffusion regime}

In the external diffusion regime, the reaction rate is determined by general diffusion laws, values of the mass 
transfer coefficient of reactants and fluid dynamic parameters. Under steady state conditions, the reaction rate for first order will be equal to the diffusion rate:

$$
r_{\text {reaction }}=r_{\text {diffusion }} \text { or } k_{\mathrm{c}} \cdot\left(C_{0}-C_{\mathrm{s}}\right)=k \cdot C_{\mathrm{s}}
$$

The surface concentration $C_{\mathrm{s}}$ is equal to:

$$
C_{\mathrm{s}}=\frac{k_{\mathrm{c}} \cdot C_{0}}{\left(k_{\mathrm{c}}+k\right)}
$$

Then the reaction rate in external diffusion regime $\left(r_{\mathrm{ed}}\right)$ is given by:

$$
r_{\text {ed }}=\frac{k \cdot k_{\mathrm{c}}}{k+k_{\mathrm{c}}} C_{0}=\frac{1}{\frac{1}{k}+\frac{1}{k_{\mathrm{c}}}} C_{0}=k_{\mathrm{eff}} \cdot C_{0}
$$

The reverse value of the effective rate constant $k_{\text {eff }}$ is equal to the sum of the kinetic $(1 / k)$ and diffusion $\left(1 / k_{\mathrm{c}}\right)$ resistances. If $k_{\mathrm{c}} \gg k$, then $C_{0} \approx C_{\mathrm{s}}$ and $k_{\mathrm{eff}} \approx k$. In this case, the reaction rate will be determined by the chemical reaction, i.e. the reaction proceeds in the kinetic regime. If $k>>$ $k_{\mathrm{c}}$, then $\mathrm{C}_{\mathrm{s}} \approx 0$ and $k_{\mathrm{eff}} \approx k_{\mathrm{c}}$. In this case, the reaction rate will be determined by the diffusion of the reactants to the catalyst surface, i.e. the reaction proceeds in the external diffusion regime.

In the external diffusion regime, all reactions proceed as first order reactions. It is characterized by the great temperature difference between catalyst surface and reaction mixture. Changes in the catalytic activity of the catalyst do not influence the reaction rate. Reaction rate depends on the linear flow rate or mixing and on the catalyst particle size. The effective activation energy under these conditions is equal to the activation energy of molecular diffusion $(4.2-12.5 \mathrm{~kJ} / \mathrm{mol})$. The influence of different factors on the reaction rate in external diffusion regime is discussed below.

Temperature - The reaction rate in external diffusion regime is proportional to the temperature. This dependence is determined by the temperature dependence on the diffusion coefficient of reactants. Therefore increasing the reaction temperature leads to a transition from kinetic to the external diffusion regime and vice versa. Due to low velocity of the heat transfer process, a large increase in the catalyst temperature can be observed, which can lead to the appearance of an autothermal regime under steady state conditions.

Pressure - The diffusion coefficient increases with the decrease of the pressure, which leads to an increase of the reaction rate in the external diffusion regime. However, the pressure also has influence on other process parameters like linear velocity, and in general, the dependence becomes more complicated.

Flow rate - The increase of the flow rate leads to increasing mass transfer processes and therefore it helps to transfer the system into the kinetic regime.

Mixing - By increasing the mixing intensity, the fluid flow becomes more turbulent and decreases the thickness of the boundary laminar layer. This means that the reaction rate is increased, and it is possible that the external diffusion influence becomes negligible under certain mixing conditions.

Catalyst particle size - The smaller the catalyst particle size is, the higher the diffusion rate is. Therefore, the higher is the reaction rate.

Catalyst particle porosity - The porosity has no influence on the reaction rate in the external diffusion regime.

Catalyst activity - In the external diffusion regime, the reaction rate is controlled by the external diffusion process. Increasing the catalyst activity does not influence the overall reaction rate. Decreasing the catalyst activity will permit the system to enter the kinetic regime. Therefore, if certain catalytic processes proceed in the external diffusion regime the type and activity of the used catalyst only play minor role.

Catalyst selectivity - The transition from kinetic to the external diffusion regime can change the reaction selectivity in both consecutive and parallel type of reactions. This is due to the increase of residence time of the reactants and products at the catalyst surface.

Catalyst poisoning and deactivation - The catalyst poisoning and deactivation do not exert any influence on the reaction rate in the external diffusion regime until the catalytic activity is decreased to such an extent that the system is transferred into the kinetic regime. Under these conditions, it is possible that the poisoning rate can be decreased due to slow diffusion to the catalyst surface.

\subsection{Heat and mass transfer in porous catalysts}

Heterogeneous catalytic reactions proceed on the surface of porous catalysts and therefore are accompanied by pore diffusion of the reactants and reaction products into and out of the catalyst grains and heat transfer process.

Pore diffusion may occur by one or more of three mechanisms: ordinary (molecular) diffusion, Knudsen diffusion, and surface diffusion. The last one has no serious impact on the kinetics of heterogeneous catalytic reactions. The general characteristics of molecular diffusion have been discussed in the previous section of this paper. Knudsen diffusion can be observed when gas density is low or pores are quite small or in both cases. Under these conditions, molecules collide with pore walls much more frequently than with one another. Knudsen diffusion is independent of pressure and is not observed in liquids. Kinetic theory provides following relation for Knudsen diffusion coefficient $\left(D_{\mathrm{k}}\right)$ in a straight round pore:

$$
D_{\mathrm{k}}=9700 \cdot r_{\mathrm{e}} \sqrt{\frac{T}{M}}
$$

where $r_{\mathrm{e}}$ is the pore radius in $\mathrm{cm}, T$ is the absolute temperature, and $M$ is the molecular weight. Since the number of molecular collisions is negligible, each component in a re- 
action mixture behaves as it is present alone.

The pore geometry of solid catalysts is poorly understood and empirical model must be used. Straight round pore with radius $r_{\mathrm{e}}$ has volume to surface ratio of $1 / 2 r_{\mathrm{e}}$. If the porous material has a total surface, $S_{\mathrm{g}}\left(\mathrm{cm}^{2} / \mathrm{g}\right)$ and average pellet density $\rho_{\mathrm{p}}\left(\mathrm{g} / \mathrm{cm}^{3}\right)$ for pore radius $r_{\mathrm{e}}$ can be obtained:

$$
r_{\mathrm{e}}=\frac{2 V_{\mathrm{g}}}{S_{\mathrm{g}}}=\frac{2 \theta}{S_{\mathrm{g}} \cdot \rho_{\mathrm{p}}}
$$

and the Knudsen diffusion coefficient for a porous solid becomes:

$$
K_{\mathrm{K}, \text { eff }}=\frac{D_{\mathrm{K}} \cdot \theta}{\tau_{\mathrm{m}}}=19400 \frac{\theta^{2}}{3 \tau_{\mathrm{m}} \cdot S_{\mathrm{g}} \rho_{\mathrm{p}}} \sqrt{\frac{T}{M}}
$$

The void fraction $\theta$ has been introduced so the flux given by $D_{\mathrm{K}, \text { eff }}$ will be based on the total cross section of porous solid, but not just the pore cross section. The tortuosity factor $\tau_{\mathrm{m}}$ accounts for both tortuous path and the effect of the varying cross section of individual pores. In many cases, the solids contain pores with broad distribution of pore radius. It means that the diffusion in pores will proceed by both Knudsen and molecular diffusion. This regime is called transition. For the effective diffusion coefficient $\left(D_{\text {eff }}\right)$ in the transition regime, one can obtain the following equation:

$$
\frac{1}{D_{\text {eff }}}=\frac{1}{D_{\mathrm{K}, \text { eff }}}+\frac{1}{D_{1,2, \text { eff }}}
$$

where $D_{1,2, \text { eff }}=D_{1,2} \cdot \theta / \tau$ is the effective diffusion coefficient for molecular diffusion.

Several critical reviews on experimental methods for evolution of catalyst effective diffusion coefficients of heterogeneous catalysts have been published recently [47-49].

\subsection{Internal diffusion regime}

In order to take account of the influence of pore diffusion on reaction rate, the effectiveness factor $\eta$ is introduced.

$$
r=\eta \cdot k \cdot C_{\mathrm{s}}^{n}
$$

where $\eta=r_{\text {obs }} / r_{\text {intr }}, r_{\text {obs }}$ is the observed reaction rate, $r_{\text {intr }}$ is the intrinsic chemical reaction rate, $C_{\mathrm{s}}$ is the concentration on the particles surface, and $n$ is the reaction order. If $\eta$ has value between 0.95 and 1 , no internal diffusion resistance is taking place.

The theoretical treatment of the problem leads to the following expression for $\eta$ as a function of Thiele modulus $\phi$ :

$$
\eta=\frac{1}{\phi}\left(\frac{1}{\operatorname{tgh} 3 \phi}-\frac{1}{3 \phi}\right)
$$

The Thiele modulus is given by the formula:

$$
\phi=R \sqrt{\frac{k \cdot C_{\mathrm{s}}^{n-1}}{D_{\mathrm{eff}}}}
$$

The Thiele modulus performs in one criterion which combines the particle size $R$ (representing the catalyst macrostructure), the effective diffusion coefficient $D_{\text {eff }}$ (repre- senting the catalysts microstructure and porosity), and $k \cdot C_{\mathrm{s}}^{n-1}$ (representing the chemical reaction characteristics). The square of the Thiele modulus is the ratio of the characteristic times for mass transport $\tau_{\text {diffusion }}$ to reaction $\tau_{\text {chemreaction }}$ :

$$
\phi^{2}=\frac{R^{2}\left(k \cdot C_{\mathrm{s}}^{n-1}\right)}{D_{\text {eff }}}=\frac{\tau_{\text {diffusion }}}{\tau_{\text {chemreaction }}}
$$

The effective depth of the reaction $H$ inside catalyst grains can be estimated by the formula:

$$
H=\gamma \sqrt{\frac{r}{C_{\mathrm{s}} \cdot D_{\text {eff }}}}
$$

where $\gamma$ is constant with the value near to unity.

The influence of different factors on the reaction rate in the internal diffusion regime is discussed below.

Temperature - The temperature dependence in the internal diffusion regime is not very strong compared with that in the kinetic regime. A catalyst operating at a low effectiveness factor will show apparent activation energy on an Arrhenius plot that is arithmetic mean of the activation energies for the diffusion process and chemical reaction.

$$
E_{\text {obs }}=0.5\left(E+E_{\mathrm{D}}\right)
$$

Temperature regime - The temperature gradients within catalyst pellets in the internal diffusion regime are, in general, more favorable than in the kinetic regime. The pellets are highly nonisothermal during the processes accompanied by high heat effect and high activation energy. This circumstance can lead to a compensation for diffusion retardation and the reaction rate in diffusion regime can be larger than that in the kinetic regime. Increasing the reaction temperature leads to a transition from kinetic to the internal diffusion regime and vice versa.

Pressure - High pressure increase the probability of transition from Knudsen diffusion to molecular diffusion. At the same time, the molecular diffusion coefficient decreases with pressure increase. The pressure influence depends upon the form of kinetic equation used to describe the reaction rate which is dependent on pressure. In general, the pressure influence cannot be predicted in advance.

Flow rate - Low flow rates help the transition from internal diffusion to kinetic regime.

Catalyst particle size - Decreasing particle size permits larger part of the grain to participate in the reaction. When the entire internal surface becomes accessible to reactant molecules, a transition to the kinetic regime is accomplished.

Catalyst porosity - Reaction rate must be increased with the increasing catalyst porosity because both Knudsen and molecular diffusion coefficients increase in larger pores.

Reaction rate - The reaction rate in diffusion regime is smaller than that in kinetic regime. The higher the reaction order is the stronger the influence of the internal diffusion 
is. At high degrees of conversion, the transition to kinetic regime becomes more probable.

Diffusion disguise of selectivity - When diffusion resistance exists inside a catalyst particle, the selectivity may change depending on the type of occurring reaction.

\subsection{Criteria for absence of diffusion limitations}

Various criteria are proposed for establishing the absence of diffusion limitations on reaction rate. Here we will give the simplest one - Weisz and Prater [50] criterion for isothermal spherical particle at effectiveness factor $\eta \geq 0.95$.

$$
\frac{r \cdot R_{\mathrm{p}}^{2}}{C_{0} \cdot D_{\text {eff }}}<\psi
$$

where $r$ is the reaction rate per unit catalyst particle volume, $R_{\mathrm{p}}$ is the catalyst particle diameter, $C_{0}$ is the reagent concentration at the catalyst surface, and $D_{\text {eff }}$ is the effective diffusion coefficient of the reagent within the catalyst particle. The value of $\psi$ depends on the reaction order in the following way: $\psi=6$ for a zero order reaction; $\psi=1$ for a first order reaction; $\psi=0.3$ for a second order reaction.

\section{Kinetics of heterogeneous catalytic reactions and catalyst deactivation}

There are several types of catalyst deactivation which are caused by different reasons like sintering, poisoning, and changes of catalytic activity due to interactions with reagents: (i) strong and irreversible adsorption of some reagents; (ii) interaction of reactants with catalytic centers; (iii) induced diffusion of lattice components towards catalyst surface; (iv) formation of coke precursors blocking the catalyst surface [51-56]. The mechanisms of catalyst deactivation are of great variety.

Kinetic descriptions of catalyst deactivation are generally two types.

(i) Inseparable deactivation. Catalyst deactivation is an inseparable part of the reaction mechanism and this process is described by special term in the kinetic equation.

$$
r=F\left(k(t), P_{i}, T, \psi\right)
$$

where $k(t)$ is the rate constant, which is a function of time $t$, $P_{i}$ is the partial pressures of reagents and/or reaction product, $T$ is reaction temperature, and $\psi$ is the term describing the catalyst deactivation.

(ii) Separable deactivation. Catalyst deactivation is described by rate model, which is uncoupled from the rate equation describing the main reaction.

$$
r=r_{\text {kin }}\left(k, P_{i}, T\right) \cdot\left(\varphi_{\mathrm{d}}\left(k_{\mathrm{d}}, P_{i}, T\right)\right)_{\tau}
$$

where $r_{\text {kin }}\left(k, P_{i}, T\right)$ is a kinetic model of main reaction at constant catalyst activity, and $\phi_{\mathrm{d}}\left(k_{\mathrm{d}}, P_{i}, T\right)_{\tau}$ is a kinetic model of the reaction of catalyst deactivation.

Recently we published a paper [57], in which most of above-mentioned issues were discussed in details. Kinetic models, which take the catalyst deactivation into account, are of great importance for practical purposes especially for catalytic processes over fast deactivating catalysts.

\section{Mathematical treatment of experimental kinetic data}

The mathematical treatment of the experimental data should pass through a number of different procedures.

\subsection{Design of the kinetic experiments}

Sequential experimental design for planning of kinetic experiments on theory gives the optimal experimental strategy $[58,59]$. However, practical application of this design meets serious difficulties due to specific characteristics of the type of reactor used in the experimental study, the computer calculations connected with the used set of reaction models, number of kinetic constants and optimization algorithms used. Discussion about these difficulties is given in Ref. [60].

\subsection{Calculating rates on the independent reaction routes}

The reaction rates on independent routes "P" are not obtained directly from the experiments. It should be stressed again that in the kinetic experiments reaction rates for every reagent " $Q$ " are measured. From this experimental data, the rates of reaction routes are calculated. In order to decrease the influence of the experimental error, it is necessary to solve the over determined system of Eq. (13) since $q>P$. The number of equations is equal to that of reagents and the number of unknowns is equal to that of reaction routes.

$$
r_{i}=\sum_{j=1}^{M_{T}}\left\|\mu_{i, j}\right\|^{T} R_{j}
$$

where $r_{i}$ is the experimental reaction rate for the $i^{\text {th }}$ reagent $(i$ $=1,2, \ldots Q), R_{j}$ is calculated reaction rates on $j^{\text {th }}$ independent route $(j=1,2, \ldots P)$, and $\left\|\mu_{i, j}\right\|^{T}$ is transposed stoichiometric matrix of the independent reaction route. Always $i>j$. Problems arising during these calculations are discussed in Refs. [61-64].

\subsection{Kinetic parameters estimation}

In order to find the values of reaction rate constants of the elementary steps, additional information is needed which can be obtained from nonsteady state kinetic measurements 
and other experimental and theoretical methods like chemisorption, quantum chemistry, and surface science methods. Parameter estimation needs application of statistical and nonlinear programming methods. Linear least square methods are not suitable for kinetic parameters estimation, since in most of the cases the kinetic models are nonlinear. It is recommendable for this aim to use nonlinear programming. Numbers of nonlinear algorithms have been proposed. The widely used methods are flexible simplex proposed by Nelder-Mead [65], Marquardt [66], and Rosenbrock [67]. The problem of confidence range of kinetic rate constants estimated from steady state experiments is discussed in Ref. [68].

The reparametrization of the rate constants proposed by Frank-Kamenetzkii [28] should be used, which allow to avoid correlation between preexponential factor and activation energy. Since the values of activation energy and preexponential factor sometimes differ in 10 orders of magnitudes, it is necessary to use normalization to make the parameter search more effective. According to Frank-Kamenetzkii reparametrization, the expression for the Arrhenius equation (Eq. (15)) is replaced by

$$
\begin{gathered}
k=k_{0}^{\prime} \exp \left(\frac{-E}{R}\left(\frac{1}{T}-\frac{1}{T_{\text {average }}}\right)\right) \text {, where } \\
k_{0}^{\prime}=k_{0} \exp \left(\frac{-E}{R T_{\text {average }}}\right)
\end{gathered}
$$

For minimization, different criteria of $E$ might be used:

(i) Sum of the squares of the deviations between experimental rate values and those predicted by a given kinetic model.

$$
W_{i}=\left(r_{i}^{\exp }-r_{i}^{\mathrm{calc}}\right) \quad E=\sum_{1}^{N} W_{i}^{2}
$$

where $N$ is the number of experiments, $r_{i}^{\text {exp }}$ and $r_{i}^{\text {calc }}$ are reaction rates of experimental and calculated by the proposed model, which are independent of routes.

(ii) Sum of the squares of the relative errors.

$$
W_{i}=\left(\frac{r_{i}^{\exp }-r_{i}^{\mathrm{calc}}}{r_{i}^{\exp }}\right) \quad E=\sum_{1}^{N} W_{i}^{2}
$$

(iii) Sum of the modules of the deviations between experimental rate values and those predicted by a given kinetic model.

$$
W_{i}=\left(\left|r_{i}^{\exp }\right|-\left|r_{i}^{\mathrm{calc}}\right|\right) \quad E=\sum_{i=1}^{N} W_{i}
$$

(iv) Minimization of the maximal deviation between experimental rate values and those predicted by a given kinetic model.

$$
W=\min \max \left(\left|r_{i}^{\exp }\right|-\left|r_{i}^{\mathrm{calc}}\right|\right) \quad E=W
$$

(v) For multiroute reactions we have

$$
E=\sum_{q=1}^{Q} \sum_{i=1}^{N}\left(W_{i}^{(q)}\right)^{2}
$$

where $Q$ is the number of stoichiometrically independent reaction routes, and $N$ is the number of experiments.

The use of particular criterion strongly depends on the distribution character the of the experimental error.

\subsection{Statistical assessment of the best reaction models}

For these aims, standard statistical procedures are used [59]. The number of experiments $(N)$ gives the freedom degree of the experimental points. The number of constants " $p$ " in the reaction rate model gives the freedom degree of the model. Residual freedom degree $N-p$ is a criterion for judging the exactness of the model. Repeating the same experiment several times, we can establish the preciseness of the experiment

$$
\sum_{i=1}^{N}\left(r_{i}^{\text {exp }}-r_{\text {average }}^{\text {exp }}\right)^{2}
$$

we can write

$$
\sum_{i=1}^{N}\left(r_{\text {average }}^{\text {exp }}-r_{i}^{\text {exp }}\right)^{2}=\sum_{i=1}^{N}\left(r_{i}^{\text {calc }}-r_{i}^{\text {exp }}\right)^{2}-\sum_{i=1}^{N}\left(r_{i}^{\text {calc }}-r_{\text {average }}^{\text {exp }}\right)^{2}
$$

which characterize the discrepancy between the model and experiment.

\section{High-throughput approach in studies of kinetics of heterogeneous catalytic reactions}

For the moment, very few HT kinetic studies dealing with massive parallel screening have been reported in the literature, but it is predicted that this domain will undergo rapid development in the near future. There are different motivations for using kinetic modeling in HT screening [69,70].

Kinetic modeling allows proper catalyst evaluation and ranking in the case of batch reactors, thus enabling the assessment of activity for very active and moderately active catalysts.

Kinetic modeling is also useful for the rational ranking of catalysts for gas-phase reactions. From the viewpoint of reaction rates, it becomes possible to extrapolate the activity of each catalyst under a single experimental condition, and therefore to establish a fair ranking scale.

Kinetic modeling can assist in the rational assessment of a catalyst-process pair. Since the formulation, each catalyst has its own optimal process condition in terms of temperature, total and partial pressures, and contact time. Any catalyst ranking based on the results obtained under a single standard testing condition will lead to a non-optimum view and a non-rational assessment. In this case, the calculation of thermodynamic and kinetic constants allows the deter- 
mination of optimum performances for each catalyst, and ultimately of the optimum catalyst-process pair.

At the process design stage, reaction kinetics must be addressed. For example, the influence of pressure, temperature, and concentrations should be determined. This can be achieved by using a rational design of experiments to screen the appropriate variables while measuring the activity and selectivities [71-73].

\section{Kinetics of catalytic reactions on nanosized catalysts}

The well known classification introduced by Boudart [74] divides the heterogeneous catalytic processes into two groups, i.e. structure-insensitive reactions and structuresensitive reactions.

The rate of structure-insensitive reaction is not significantly changed when the particle size of active components in supported catalysts of the available crystal planes is changed. This means that their activities depend only upon the total number of surface metal atoms. Typical examples are $\mathrm{CO}$ oxidation and hydrogenation of ethylene or benzene.

A catalytic reaction said to be structure-sensitive if its rate is changed markedly when metal dispersion changes and crystal plane or defect structures take place. Typical examples are hydrogenolysis of ethane and synthesis of ammonia.

The problem of structure-sensitivity now becomes a topical issue because of very intensive developments in the area of nanocatalysis. The influence of nanosized particles on kinetic behavior of catalysts is still not well developed area, which needs further study.

Recently, in order to account the influence of nanosized catalysts on kinetics of heterogeneous catalytic reactions, Parmon $[75,76]$ proposed a thermodynamic approach based on the concept that the chemical potential $\mu_{\mathrm{r}}$ of a metal particle with a radius of curvature $r$ is different from chemical potential in a metal particle of an infinite size (bulk-like) $\mu_{\infty}$ and they are related in the following way:

$$
\mu_{\mathrm{r}}=\mu_{\infty}+\frac{2 \sigma V_{\mathrm{M}}}{r}=\mu_{\infty}+\delta(r)
$$

where $\sigma$ is the surface free energy of the metal, $V_{\mathrm{M}}$ is the atomic volume of the bulk metal, and $\delta(r)$ is the increment of chemical potential. The key factor in thermodynamic analysis is the description of the influence of nanosized particles on the chemical potential of the active phase (e.g. clusters supported on a carrier). Estimation suggests that catalyst particles of 100 atoms could be treated as a continuous phase and thus the application of standard thermodynamic approaches is possible.

It is assumed that the changes of chemical potential induced by the change of nano cluster size are dependent on the presence or absence of an adsorbate. Therefore, the decrease of the nano cluster size rather than changes in morphology is the reason for the variation of chemical potential of the active phase due to the excessive surface energy. Adsorption on nano clusters which brings changes in the Gibbs energy should also account for the chemical potential variations. Obviously, for large catalyst clusters the contribution of surface excess energy can be neglected.

Murzin [77-79] has applied the thermodynamic analysis of Parmon to the kinetics of the catalytic processes described by different reaction mechanisms. His approach is based on the Temkins theory for kinetics on nonuniform catalyst surfaces. He accepted that when the alterations of the chemical potential of nano clusters are the same in comparison with bulk metal, they were independent of surface coverage (e.g. bare surface or occupied with reactants). Since differences in the activation energy between edges and terraces are well recognized [80,81], the differences in reactivity between edges and terraces are supposed to be responsible for cluster size effects. The ratio between edges and terraces can be calculated for cubo-octahedral clusters which seem to be approximately proportional to the reciprocal value of the cluster diameter.

In the classical approach to structure-sensitive heterogeneous catalytic reactions, the surface energy excess is not taken into account. The theoretical analysis performed by Murzin is addressed to the impact of the changes in chemical potential upon adsorption. Two-step and Christiansen sequence, as well as Langmuir-Hinshelwood mechanisms were considered. It was demonstrated that for catalytic reactions carried out over nanoparticles of small size (few nanometers), kinetic equation, kinetic constants, and kinetic reaction orders can be different from those obtained for large (tens of nanometers) nano clusters. The developed approach is illustrated by comparing experimental data of catalytic activity with the average particle size for Fischer-Tropsch synthesis over cobalt loaded on carbon nano fibers, as well as for crotonaldehyde hydrogenation over gold loaded on $\mathrm{TiO}_{2}$. Selectivity analysis on metal dispersion was performed for the case of parallel and consecutive reactions, demonstrating that significant changes could be anticipated in the latter case, while only minor dependence of selectivity on metal crystallites is expected for the former case even if the activity displays substantial structure sensitivity. Provided examples suggest that thermodynamic analysis could be invoked for the explanation of nano kinetics, e.g. structure sensitivity and other kinetic regularities dependence on the size of catalytic nano clusters.

To illustrate how the approach of Murzin is accounting for the influence of nanosized particles, we shall use the two-step mechanism with two kinetically significant steps $[82,83]$ as an example, implying that one of the several sur- 
face intermediates is the most abundant, while all the others are present on the surface at much inferior concentration levels, which is widely applied in the literature to explain the heterogeneous catalytic kinetics:

$$
\begin{gathered}
\mathrm{Z}+\mathrm{A}_{1} \leftrightarrow \mathrm{ZI}+\mathrm{B}_{1} \\
\mathrm{ZI}+\mathrm{A}_{2} \leftrightarrow \mathrm{Z}+\mathrm{B}_{2} \\
\mathrm{~A}_{1}+\mathrm{A}_{2}=\mathrm{B}_{1}+\mathrm{B}_{2}
\end{gathered}
$$

where $A_{1}$ and $A_{2}$ are reactants, $B_{1}$ and $B_{2}$ are products, $Z$ is the surface site, and ZI is ISC. The reaction rate for this mechanism is given by the following equation:

$$
r=\frac{k_{1} P_{\mathrm{A}_{1}} k_{2} P_{\mathrm{A}_{2}}-k_{-1} P_{\mathrm{B}_{1}} k_{-2} P_{\mathrm{B}_{2}}}{k_{1} P_{\mathrm{A}_{1}}+k_{2} P_{\mathrm{A}_{2}}+k_{-1} P_{\mathrm{B}_{1}}+k_{-2} P_{\mathrm{B}_{2}}}
$$

where $P_{\mathrm{A}_{1}}$, etc., is partial pressure, and $k_{i}$ is the kinetic constant.

For kinetics over nanosized catalysts, Eq. (91) becomes:

$$
r_{\text {nanosized }}=\frac{\left(k_{1} P_{\mathrm{A}_{1}} k_{2} P_{\mathrm{A}_{2}}-k_{-1} P_{\mathrm{B}_{1}} k_{-2} P_{\mathrm{B}_{2}}\right) \mathrm{e}^{\frac{(1-2 \alpha) \chi}{d_{\text {cluster }}}}}{\left(k_{1} P_{\mathrm{A}_{1}}+k_{2} P_{\mathrm{A}_{2}}\right) \mathrm{e}^{\frac{-\alpha \chi}{d_{\text {cluster }}}}+\left(k_{-1} P_{\mathrm{B}_{1}}+k_{-2} P_{\mathrm{B}_{2}}\right) \mathrm{e}^{\frac{(1-\alpha) \chi}{d_{\text {cluster }}}}}
$$

where rate constants instead of $k_{i}$ are used, which are given by the expression:

$$
k_{i, \text { nanosized }=} k_{i} \mathrm{e}^{\frac{\alpha \chi}{d_{\text {cluster }}}}
$$

where $\chi$ is accounting for the changes the thermodynamic potential due to adsorption on nano clusters.

$$
\chi=\frac{\left(\Delta G_{\text {ads, angles }}-\Delta G_{\text {ads, terraces }}\right)}{R T}
$$

Thus, in general evaluation of the cluster size effect should be combined with a proper kinetic analysis when a kinetic equation is established in a reliable way. In any case, the value of parameter $\alpha$ is close to 0.5 in heterogeneous catalytic reactions, while estimated value of $\chi$ leads to a substantial difference between adsorption on edges and terraces, e.g. $\Delta G_{\text {ads,edges }}-\Delta G_{\text {ads,terraces }}$ is above $55 \mathrm{~kJ} / \mathrm{mol}$. Even a larger difference between step-edge sites and terraces of 116 $\mathrm{kJ} / \mathrm{mol}$ has been reported recently in Ref. [84].

In the papers [85-87] the authors discussed an approach for identifying how site types involving different nanostructures in multifunctional catalysts would be affected by factors causing catalyst deactivation.

Under the assumption that the catalytic clusters involve internal sites, one can distinguish the Z-type sites as $Z_{1}$-type - single internal sites, and $Z_{m}$ - catalytic clusters requiring adjacent atoms $(m)$. In view of the finite number of surface metal atoms involved within a particular active phase nanostructure, it is reasonable, when estimating the number of catalytic clusters per active phase $\left(N_{\mathrm{Z}}\right)$, to take into consideration the probability $\mu_{m}$ that surface atoms

( $m$ ) are properly located to construct a catalytic cluster. In the simplest case, the number of catalytic clusters
$\left(N_{Z_{m}}^{0}\right)$ within an active phase island on the fresh catalyst will depend on the number of single sites $N_{Z}^{0}$ located within the island, the number of surface atoms $(m)$ constructing the cluster, and the probability $\mu_{m}(\rho)$ :

$$
N_{Z m}^{0}=k_{m} \frac{1}{(m)} \mu_{m}(\rho) N_{Z}^{0}
$$

Correspondingly, the overall reaction rate $r_{\mathrm{ov}}$ may be presented as:

$$
\begin{gathered}
r_{\mathrm{ov}}=r_{Z_{1}}+r_{Z_{m}}+r_{Y}=k_{Z_{1}} \cdot f_{Z_{1}}\left(P_{j}, T\right) \cdot \phi_{Z_{1}}+ \\
k_{Z_{m}} f_{Z_{m}}\left(P_{j}, T\right) \phi_{Z_{m}}+k_{Y} f_{Y}\left(P_{j}, T\right) \cdot \phi_{Y}
\end{gathered}
$$

where the terms $r_{Z_{1}}$ and $r_{Z_{m}}$ distinguish the rates of routes facilitated by single internal sites and catalytic clusters, and the terms $f_{Z_{1}}\left(P_{j}, T\right)$ and $f_{Z_{m}}\left(P_{j}, T\right)$ stand for the corresponding kinetic regularities.

In concern of the catalyst activity attributed to the internal sites, a differentiation is needed between reactions catalyzed by single sites and catalytic clusters. The activity function of the latter depends on the probability $\left(\mu_{m}\right)$, which in turn may change more or less in the course of propagation of the coke layer:

$$
\begin{gathered}
\mathrm{d} \phi_{Z_{m}}=\frac{k_{m}}{(m)}\left\{\left(\mu_{m}\right) \mathrm{d} N_{Z_{m}}+N_{Z m}\left(\mathrm{~d} \mu_{m}\right)\right\}= \\
\frac{\kappa_{m}}{(m)}\left\{\left(\mu_{m}\right)\left(\frac{2}{\xi}\right)\left(\frac{\rho}{\xi}\right)+\left(\frac{\rho}{\xi}\right)^{2}\left(\mathrm{~d} \mu_{m}\right)\right\} \mathrm{d} \rho
\end{gathered}
$$

The Eq. (97) stands for the coking-caused decrease of the catalyst activity in regard of the structure-sensitive reactions, which are realized through the participation of multi-center intermediates. Considering Eq. (96), the selectivity for such a reaction can be presented as:

$$
S_{Z_{m}}=\frac{k_{Z_{m}} f_{Z_{m}}\left(P_{j}, T\right) \phi_{Z_{m}}}{k_{Z_{1}} f_{Z_{1}}\left(P_{j}, T\right) \phi_{Z_{1}}+k_{Z_{m}} f_{Z_{m}}\left(P_{j}, T\right) \phi_{Z_{m}}+k_{Y} f_{Y}\left(P_{j}, T\right) \phi_{Y}}
$$

It is an essential point that in the course of coke build, the probability $\mu_{m}$ for availability of single sites $(\mathrm{m})$ in proper proximity and configuration may decrease in different modes, stipulating various effects on the selectivity in regard of the structure-insensitive and structure-sensitive reactions. The system may come to a point of terminating the action of catalytic clusters, if the number and density of single sites contained in the active phase island drops to a critical level at which the probability that $(m)$ of them may be close enough to form a cluster approaching zero. Under such conditions, a stepwise change in the selectivity in favor of the structure-insensitive reaction may be expected.

The application of various criteria for operation conditions of photocatalytic reactors using nanosized photocatalyst particles is presented in Ref. [88]. 


\section{Catalytic processes under nonsteady state regime}

Most of the industrial catalytic processes proceed at steady state regimes over catalysts with constant catalytic activity. Processes included in this group are carried out over catalysts with long lifetime from several months to several years. Typical characteristic for these processes is that they are carried out under well-established steady state conditions and catalytic activity of the catalysts is practically constant during the certain period of time.

For catalytic processes proceeding under nonsteady state regime and constant temperature over catalyst with changeable catalytic activity by the full kinetic model of given, catalytic process should consist of the following autonomous differential equations:

$$
\begin{gathered}
r=\|A\|_{c} \cdot f(c, x, \alpha, T) \\
\frac{\mathrm{d} x}{\mathrm{~d} t}=\|A\|_{x} \cdot f_{1}(c, x, \alpha, T) \\
\frac{\mathrm{d} \alpha}{\mathrm{d} t}=f_{2}(c, x, \alpha, T)
\end{gathered}
$$

where $\|A\|_{c}$ and $\|A\|_{x}$ are stoichiometric matrixes for reagents and ISC, $c$ is a vector of reagent concentrations, $x$ is a vector of ISC concentrations, $\alpha$ characterizes the catalyst surface state and composition, and $T$ is the reaction temperature. If catalyst activity is constant then $\mathrm{d} \alpha / \mathrm{d} t=0$. At constant temperature, Eq. (99) describes the dependence of reaction rate on the changes of $c, x$, and $\alpha$; Eq. (100) describes the changes of the concentrations of ISC with astronomical time as a function of $c, x$, and $\alpha$; and Eq. (101) describes the changes of the catalyst state with astronomical time as a function of $c, x$, and $\alpha$. The solution of the Eqs. (99)-(101) will describe the behavior of the catalyst in nonsteady state.

From point of view of the reaction mechanism, in general, the measured reaction rates at nonsteady state conditions should be equal to the rates of some of the elementary steps, which are different from the limiting reaction steps at steady state regime. The reasons for the deviation from steady state regimes are numerous: presence of redox cycles in catalyst surface, presence of nonlinear elementary steps in the reaction mechanism, changes of reaction mechanism due to the changes of conversion degree, catalyst re-crystallization, catalyst deactivation, and catalyst poisoning, etc. Most of these factors acts spontaneously and are part of the properties of the system catalyst-reaction media. As a result, different phenomena are observed like multiple steady states, oscillating reactions, chaotic behavior, and heat explosion, etc.

Development of kinetic models of non-stationary catalytic processes is a difficult task. These models should account for the following factors: the rates of the elementary chemical transformations, the changing rates of reactant composition, the changing rates of catalyst activity, and diffusion of the reacting species in the catalyst pores, etc. The investigation of the dynamics of the catalytic process i.e. the evolution of the heterogeneous catalytic reactions gives the information, which is used for the studies and development of the dynamics and stability of the catalytic reactors and in turn which determine the optimal starting, transition, and operation regimes [88]. The problems of long-term stability of catalytic processes and possibility for transition to chaotic behavior due to macroscopic fluctuations are discussed in Ref. [89]. The conclusions of this paper are experimentally proved by studying the fractal power spectrum at catalytic oxidation of $\mathrm{HCOOH}$ over Pd catalyst $[90,91]$

Recently, a review devoted to the application of dynamic methods in catalytic kinetics has been published [92].

In some cases, heterogeneous catalytic processes are realized in big scale industrial units using nonsteady state technologies [93].

\section{Studies of the kinetics of heterogeneous catalytic processes in industrial practice}

The studies in the field of kinetics of heterogeneous catalytic reactions are an indispensable step of the theoretical and applied investigations on catalysis. They serve as fundamentals upon elucidation of the mechanism of a given heterogeneous catalytic reaction and contribute essentially to the elucidation of the catalyst behavior in the course of its preparation, exploitation, and regeneration. The reaction kinetics serves as a basis of methods development for catalytic properties tests during modeling and selection of optimal catalysts and optimization of catalytic reactors. For these reasons, the development of theory and practice in catalysis is inconceivable without performing extensive kinetic investigations. The determination of catalytic activity, selectivity, and lifetime of an industrial catalyst for a certain catalytic process is a difficult, expensive, and time-consuming task [72]

A great number of materials with different nature possess catalytic activity in one or more catalytic reactions. However, only a few of them are appropriate for preparation of industrial catalysts. There is a substantial difference between catalytic active materials and industrial catalysts. The catalytic active material should be regarded as precursor of an industrial catalyst, which has to undergo a number of transformations and treatments in order to become a real industrial catalyst. The catalytic active materials usually possess catalytic properties (activity and selectivity) but do not display the complex of properties, which are required for the industrial catalysts such as mechanical and physical 
characteristics, resistance to the poisons, and so on. The effective industrial catalysts are products, which possess very special complex of different properties:

- High catalytic activity;

- High selectivity;

- Proper pore structure;

- High resistance to deactivation and catalytic poisons;

- Long lifetime;

- Easy regeneration;

- Low operational and light-off temperature;

- High thermal stability;

- High thermal conductivity;

- High mechanical strength;

- High resistance to attrition;

- Low price.

It is impossible to prepare an industrial catalyst, which possesses all the above-mentioned properties to the highest degree. In order to obtain optimal performance in the industrial units it is always necessary to make a compromise between these different options.

Every industrial catalyst offered on the market should be together with diverse information about:

- Reaction kinetics and kinetic model;

- Catalytic activity and selectivity of the catalyst;

- Regimes for the catalyst pretreatment;

- Kinetics of catalyst deactivation;

- Regimes for catalyst regeneration;

- Lifetime of the catalyst;

- Safety transition regimes in cases of industrial accident;

- Economy of the process.

Most of the above information can be obtained by studying the reaction kinetics.

Effective application of the existing catalysts and development of new types of catalysts need precise and fast information about their catalytic properties and kinetic behavior.

Kinetic models have wide application for solving practical problems in industry. Table 1 gives idea about use of kinetics models (in percentage from total use) from different companies in their activities (Chemical Engineering News, 1996). As can be seen from the Table 1 three major areas of applications of kinetic models in industry are evaluated: (i) Process development; (ii) Catalyst development; and (iii)
Process optimization. If we try to decipher these main areas to particular topics, we can list the most important of them:

- Comprehensive kinetic model development based on detailed mechanism studies;

- Finding the best regimes of the catalyst pre-treatment in order to obtain catalyst with maximal activity and selectivity;

- Design of catalyst grains (pellet, extrusions, or granules) and studies the influence of heat and mass transfer processes on catalyst performance;

- Kinetics of catalyst deactivation processes and estimation of the regimes for catalyst regeneration;

- Estimation of the lifetime of the catalyst proposing criteria for forecasting the catalyst lifetime;

- Safety transition regimes in cases of industrial accident.

\section{Kinetics of industrial petrochemical catalytic processes}

In order to illustrate what have been set forth in the previous parts of the paper, we will discuss the results of building models for two industrial petrochemical processes, i.e. hydrogenation of nitrobenzene to aniline and epoxidation of ethylene.

\subsection{Kinetics of the hydrogenation of nitrobenzene to aniline}

Gas phase catalytic hydrogenation of nitrobenzene is a principle industrial method for aniline production. The copper catalyst supported on kieselguhr is used. Under the conditions used in industry, the catalyst is steady deactivated with typical lifetime of about $3000 \mathrm{~h}$. In view of this, the effects of deactivation and mass transfer should be thoroughly considered during the development kinetic models in the process. Therefore, at the beginning we will study the mass transfer of the pellets at different steps of their use.

\subsubsection{Design of catalyst pellets}

Catalytically active materials can be used as the industrial catalysts after shaping. By selecting the proper shape and grains structure, the optimization of the catalysts can control

Table 1 Use of kinetic models (in percentage from total use) by different type companies

\begin{tabular}{lcccc}
\hline Type of company & Trouble-shouting & $\begin{array}{c}\text { Process } \\
\text { development }\end{array}$ & $\begin{array}{c}\text { Catalyst } \\
\text { development }\end{array}$ & $\begin{array}{c}\text { Process } \\
\text { optimization }\end{array}$ \\
\hline Chemical & 14 & 29 & 22 & 27 \\
Oil processing & 10 & 27 & 28 & 33 \\
Catalyst producer & 16 & 13 & 47 & 14 \\
Engineering & 2 & 55 & 15 & 27 \\
\hline
\end{tabular}


the catalyst performance in industrial reactors as well as the heat and mass transfer in the grains. Obviously, design of technical catalysts requires not only the experience in preparing catalysts, but also the knowledge about reactor technology, kinetics of the reaction, and transfer processes and the hydrodynamics [94].

Under working conditions, industrial catalysts undergo considerable changes on the properties of their chemical composition, crystal morphology, catalyst's texture, etc. These changes have strong influence on the catalytic properties as well as the heat and mass transfer processes in the catalyst grains. In order to get optimal performance the catalyst should be used in properly designed catalytic reactor and with properly designed grains. By studying the kinetics, catalytic activity and morphological properties of the grains at different steps of their lifetime, the effective information about the optimization of grain size and shape can be obtained. The basic parameters related to the mass transfer in the catalyst grain are the effective diffusion coefficients of reagents and tortuosity factor of catalyst pores.

We will review about the development of pellets of industrial copper catalyst used in the processes of hydrogenation of nitrobenzene to aniline by studying the kinetics, effective diffusion coefficients, and tortuosity factors of catalysts pores at different stages of the catalyst application.

The catalyst contained $50 \mathrm{wt} \% \mathrm{Cu}$ precipitated on activated Kieselghur promoted with chromia and nickel. Commercial cylindrical pellets were of $5 \mathrm{~mm} \times 5 \mathrm{~mm}$ size, 1.25 $\mathrm{kg} / \mathrm{L}$ bulk weight, and $100 \mathrm{~kg} / \mathrm{cm}^{2}$ crushing strength. The BET surface area of the catalyst was $119 \mathrm{~m}^{2} / \mathrm{g}$. The pore volume and mean pore radius, measured by mercury porosimetry, were $0.23 \mathrm{~cm}^{3} / \mathrm{g}$ and $4.91 \times 10^{-7} \mathrm{~cm}$, respectively.

Before the use of the freshly prepared $\mathrm{Cu}$, hydrogenation catalyst should be reduced in the industrial reactor under the stream of hydrogen and nitrogen mixture at elevated temperatures. During this procedure, the active phase of the catalyst formed and at the same time, changes in its texture and mechanical properties were observed. The lifetime of the catalysts was $3000 \mathrm{~h}$. The deactivation of the catalyst is mainly due to the presence of non-reacted nitrobenzene in reaction mixture. For that reason, the catalyst is discharged from the reactor at the moment when the aniline at the reactor contains $0.01 \%$ nitrobenzene. Therefore, the discharged catalyst is only partially deactivated.

The diffusion characteristics were determined by chromatographic method (CM) and dynamic method of Wicke-Kallenbach (DMWK). The following catalyst samples were used:

(i) Freshly prepared catalyst in oxide form;

(ii) Freshly prepared catalyst reduced in the diffusion cell or in the chromatographic column;

(iii) Catalyst samples after $1000 \mathrm{~h}$ use in industrial reac- tor;

(iv) Discharged catalyst samples after $3000 \mathrm{~h}$ use in the industrial reactor.

During the past years, variants of the chromatographic method for measurement of the transfer parameters have been developed [95-99], but all of them are based on the theory, which correlated to the Kubin-Kucera model [100-102]. According to this model, the moments of chromatographic peak at the column outlet are correlated with the mass transfer parameters: the axial dispersion coefficient $E_{\mathrm{a}}$, the external mass transfer coefficient $k_{\mathrm{f}}^{\text {ext }}$, the internal mass transfer coefficient $k_{\mathrm{f}}^{\mathrm{int}}$, and the effective diffusion coefficient in the catalyst pores $D_{\text {eff }}$.

The most important advantage of the DMWK [103-111] is to avoid the influence of side effects, such as an axial dispersion and external diffusion. By using DMWK, it is easier to discuss the influence of the pelletizing conditions and pellet size on the diffusion characteristics of catalyst. In most cases, the most reasonable results are obtained when both methods are used.

From data presented in Tables 2, it is evident that the DMWK gives always higher values for $D_{\text {eff }}$ than the CM. Nevertheless, there exists good agreement between values of $D_{\text {eff }}$ obtained by both methods. With increasing the temperature of measurements, the difference between results obtained by two methods is not obvious.

The average deviation for values obtained by Wicke-Kallenbach method is $13.9 \%$, while for the chromatographic method the deviation is $36.7 \%$.

The tortuosity factor $\tau$ is an important parameter for solid catalysts. It is a ratio of the distance between a diffusing species traveling on the average between two points inside the pellet, and the geometric distance between these two points. The theoretical predictions give values of 1.41 for loosely packed materials [42].

On theory $\tau$ is a independent property of the diffusing gases and is only dependent on the texture of the porous solid [112]. The $\tau$ values in Table 2 are much higher than the theoretical value 1.41 , which are not unusual for the industrial catalysts. For the tortuosity factor reported in the literature [113] the predominant values are between 2 and 4 . According to Wang and Smith [114] the average $\tau$ value for industrial catalysts should be around 8 when Knudsen and molecular diffusions are taken into account. For many industrial catalysts higher $\tau$ values are reported as 11.1 for high temperature WGSR catalyst, 12.5 for ammonia synthesis catalyst [115], 4.53 for diatomaceous earth [116], and 5-10 for spherical activated alumina [117].

For CM, $\tau$ was estimated based on the entire number of experiments conducted at given temperature. This contributed to a more correct determination of the tortuosity factor of catalyst pores. 
Table 2 Influence of the reduction and deactivation on the values of $D_{\text {eff }}$ obtained from chromatographic and Wicke-Kallenbach methods

\begin{tabular}{|c|c|c|c|c|c|c|}
\hline \multirow{2}{*}{ Catalyst } & \multirow{2}{*}{ Temperature $(\mathrm{K})$} & \multicolumn{3}{|c|}{ Chromatographic method } & \multicolumn{2}{|c|}{ Dynamic method of Wicke-Kallenbach } \\
\hline & & $D_{\mathrm{eff}} /\left(\mathrm{cm}^{2} / \mathrm{s}\right)$ & $\tau$ & $r_{\mathrm{e}} /\left(10^{-7} \mathrm{~cm}\right)$ & $D_{\mathrm{eff} f} /\left(\mathrm{cm}^{2} / \mathrm{s}\right)$ & $\tau$ \\
\hline \multirow[t]{3}{*}{ Fresh, non-reduced } & 293 & 0.32 & 7.04 & 1.93 & 0.36 & 8.16 \\
\hline & 353 & 0.39 & 5.21 & 1.99 & 0.41 & 5.59 \\
\hline & 433 & 0.46 & 3.89 & 2.06 & 0.49 & 4.97 \\
\hline \multirow[t]{3}{*}{ Fresh, reduced } & 293 & 0.68 & 5.89 & 2.25 & 1.55 & 3.42 \\
\hline & 353 & 2.03 & 4.43 & 2.32 & 2.54 & 1.74 \\
\hline & 433 & 2.89 & 3.31 & 2.40 & 3.42 & 1.44 \\
\hline \multirow[t]{3}{*}{ Deactivated at $1000 \mathrm{~h}$} & 293 & 0.54 & 4.12 & 1.66 & 0.76 & 3.04 \\
\hline & 353 & 1.63 & 3.10 & 1.73 & 1.89 & 1.39 \\
\hline & 433 & 2.31 & 2.32 & 1.79 & 2.55 & 1.18 \\
\hline \multirow[t]{3}{*}{ Deactivated at $3000 \mathrm{~h}$} & 293 & 0.32 & 2.84 & 1.28 & 0.38 & 2.46 \\
\hline & 353 & 0.96 & 2.14 & 1.34 & 1.08 & 1.01 \\
\hline & 433 & 1.36 & 1.60 & 1.43 & 1.55 & 0.79 \\
\hline
\end{tabular}

The tortuosity factor, determined by the Wicke- Kallenbach method, is estimated on strictly defined conditions (temperature, gas pairs). In Table 2, average values obtained with different gas pairs are reported.

After catalyst reduction, increase in $D_{\text {eff }}$ is observed. The changes of the $D_{\text {eff }}$ values are temperature dependent. The higher measurement temperature is, the larger is the difference between the $D_{\text {eff }}$ for oxide and reduced forms.

The effect of different reaction systems on transfer characteristics of the investigated samples should be taken into account when kinetics is studied in modeling reactor and on the working conditions of the industry. From the data in Table 2, besides the measurements of the effective diffusion coefficients of reagents and tortuosity factor of catalyst pores many other factors should be taken into account. The most important factors are: (i) The state of the catalyst sample, in which the catalyst is supposed to be used; (ii) The temperatures, at which the studies are performed should correspond; (iii) Catalyst deactivation by coking and high reaction temperatures resulting in decreasing of the tortuosity factor which means catalyst surface area become easily accessible.

\subsubsection{Kinetic models of the reaction of nitrobenzene hydrogenation to aniline}

The process of aniline production is carried out at steadily deactivated catalysts. Therefore, it is necessary to obtain kinetic information about process behavior at different state of the catalyst. The results are given below.

\subsubsection{In the absence of diffusion limitations and deactivation}

The experimental data [118] can be described by the following kinetic scheme, in which steps 1) and 5) are reversible, and the other three steps are irreversible:
1) $\mathrm{C}_{6} \mathrm{H}_{5} \mathrm{NO}_{2}+[\mathrm{K}]=\left[\mathrm{C}_{6} \mathrm{H}_{5} \mathrm{NO}_{2} \mathrm{~K}\right]$

2) $\left[\mathrm{C}_{6} \mathrm{H}_{5} \mathrm{NO}_{2} \mathrm{~K}\right]+\mathrm{H}_{2} \rightarrow\left[\mathrm{C}_{6} \mathrm{H}_{5} \mathrm{NOK}\right]+\mathrm{H}_{2} \mathrm{O}$

3) $\left[\mathrm{C}_{6} \mathrm{H}_{5} \mathrm{NOK}\right]+\mathrm{H}_{2} \rightarrow\left[\mathrm{C}_{6} \mathrm{H}_{5} \mathrm{NHOHK}\right]$

4) $\left[\mathrm{C}_{6} \mathrm{H}_{5} \mathrm{NHOHK}\right]+\mathrm{H}_{2} \rightarrow\left[\mathrm{C}_{6} \mathrm{H}_{5} \mathrm{NH}_{2} \mathrm{~K}\right]+\mathrm{H}_{2} \mathrm{O}$

5) $\left[\mathrm{C}_{6} \mathrm{H}_{5} \mathrm{NH}_{2} \mathrm{~K}\right]=\mathrm{C}_{6} \mathrm{H}_{5} \mathrm{NH}_{2}+[\mathrm{K}]$

The following kinetic model is developed

$$
r_{0}=\frac{k_{1} P_{\mathrm{NB}} P_{H_{2}}}{1+k_{2} P_{\mathrm{NB}}}
$$

The mean deviation of the reaction rates between experimental values and calculated ones was $20.3 \%$ based on the constants $k_{1}^{0}=2.4 \times 10^{-3}, E_{1}=43300 \mathrm{~J} / \mathrm{mol} ; k_{2}^{0}=6.8 \times$ $10^{-4}, E_{2}=34500 \mathrm{~J} / \mathrm{mol}$.

\subsubsection{Effects of the catalyst deactivation in the kinetic region}

The main reason for catalyst deactivation showed by experimental data is the presence of non-reacted nitrobenzene in the reaction mixture at the reactor exit whilst the partial pressure of hydrogen acts as a factor in decreasing the deactivation. The deactivation process can be described by adding two more steps given below to the reaction scheme (102).

6) $\left[\mathrm{C}_{6} \mathrm{H}_{5} \mathrm{NO}_{2} \mathrm{~K}\right]+\left[\mathrm{C}_{6} \mathrm{H}_{5} \mathrm{NHOHK}\right] \rightarrow[\mathrm{RK}]+n \mathrm{H}_{2}$

7) $m[\mathrm{RK}] \rightarrow$ coke where [RK] is a specie of a coke precursor and $m$ and $n \geq 2$.

Considering the catalyst deactivation in the kinetic region, the kinetic model derived by the reaction scheme (102) with the addition of reaction steps (94) is given below [119].

$$
r_{\tau}=\frac{k_{1} P_{\mathrm{NB}} P_{\mathrm{H}_{2}}}{1+k_{2} P_{\mathrm{NB}}}\left(1-K^{*}\left[\left(\frac{P_{\mathrm{H}_{2}}}{P_{\mathrm{NB}}}\right)_{\tau}-\left(\frac{P_{\mathrm{H}_{2}}}{P_{\mathrm{NB}}}\right)_{\tau_{0}}\right]\right)
$$

where $k_{1}$ and $k_{2}$ are taken from Eq. (103), and deactivation constant $K^{*}=10.34$. The accuracy of this model in relation to the experimentally measured reaction rates is $13.7 \%$. 


\subsubsection{Effects of the intraparticle resistance}

Under these conditions, the reaction model has the form

$$
r_{\mathrm{dif}}=\frac{k_{1}^{\mathrm{dif}}}{R\left(2 D_{\mathrm{eff}} P_{\mathrm{H}_{2}}\right)^{0.5}}\left\{\frac{1}{k_{2}^{\mathrm{dif}}}\left[P_{\mathrm{NB}}-\frac{1}{k_{2}^{\mathrm{dif}}} \ln \left(1+k_{2}^{\mathrm{dif}} P_{\mathrm{NB}}\right)\right]\right\}^{0.5}
$$

where $k_{1}^{\text {dif }}$ and $k_{2}^{\text {dif }}$ are the deactivation parameters. The Eq. (106) conformed to the experimental data with accuracy of $11.8 \%$.

\subsubsection{Effects of the intraparticle resistance and catalyst deactivation}

This model takes the influence of both catalyst deactivation and mass transfer limitations into account:

$$
\begin{gathered}
r_{\text {dif,deact }, \tau}=\frac{k_{1}^{\text {dif,deact }}\left(D_{\text {eff }} P_{\mathrm{H}_{2}}\right)^{0.5}}{R_{\mathrm{g}}}\left\{\frac { 1 } { k _ { 2 } ^ { \text { dif,deact } } } \left[P_{\mathrm{NB}, \tau}-\right.\right. \\
\left.\left.\frac{1}{k_{2}^{\text {dif,deact }}} \ln \left(1+k_{2}^{\text {dif,deact }} P_{\mathrm{NB}, \tau}\right)\right]\right\}^{0.5} \cdot\left[1-K^{*}\left(1-\frac{P_{\mathrm{NB}, \tau}}{P_{\mathrm{NB}, 0}}\right)\right]
\end{gathered}
$$

where $P_{\mathrm{NB}, 0}$ and $P_{\mathrm{NB}, \tau}$ are the nitrobenzene partial pressures at the beginning of the reaction and at the moment $\tau, K^{*}$ is the deactivation constant, $k_{1}^{\text {dif,deact }}$ and $k_{2}^{\text {dif,deact }}$ are kinetic constants, and $R_{\mathrm{g}}$ is the catalyst particle diameter. The Eq. (107) conformed to the experimental data with accuracy of $6.9 \%$.

The four derived kinetic models that are describing the same catalytic process under different reaction conditions differ substantially. According to model (105) description, the reaction in kinetic region is combined with catalyst deactivation: the increased partial pressures of hydrogen prevent catalyst deactivation while the increased partial pressures of nitrobenzene increase the catalyst deactivation. The model (106) describes hydrogenation of nitrobenzene in the internal diffusion regime. In this regime, the deactivation reaction rate depends upon the partial pressure of nitrobenzene. This explains why the catalyst deactivation is more intensive in the presence of diffusion limitations.

The proposed set of kinetic models permits to describe the hydrogenation process under different reaction conditions.

\subsubsection{Accelerated method for catalyst lifetime estimation}

The most time-consuming step during the design and development of industrial catalysts and the quality-checking procedures is the estimation of catalyst lifetime. The express method for one-day laboratory test is proposed for prognosis of the activities and lifetime of the catalyst for gas phase hydrogenation of nitrobenzene to aniline [72,120,121].
The method is based on the deactivation behavior of the catalysts under different reaction conditions, including extreme condition of very high liquid hourly space velocity (LHSV), and it permits to predict the industrial lifetime and performance using the data obtained from one-day test under laboratory conditions.

It is well known that different LHSV values determine different lifetime of the catalyst. The curve of LHSV/lifetime is an individual property of a given catalyst. It has reproducible shape characteristics for any single catalyst, which has to be established experimentally. Any point from the LHSV/lifetime curve can be used for lifetime estimation. It is clear that the aim of expressing lifetime estimation is to obtain reliable result for a period of time as short as possible. Therefore, it is expedient to use very high LHSV. Under these conditions, serious problems with heat and mass transfer may appear. In order to describe this behavior of the catalyst, the Eq. (107) is used.

The efficiency of the proposed method is tested and elaborated in different industrial units for catalysts production and exploitation.

\subsection{Kinetics of ethylene epoxidation}

Ethylene oxide is an important intermediate in the manufacture of ethylene glycol, surfactants, polymers, and many other products. It is produced by selective oxidation of ethane by oxygen or air over silver catalysts. The process proceeds in two routes with high selectivity, which is increased additionally by adding small quantity of dichloroethane as a selectivity promoter. The lifetime of the catalysts used in industry is more than 3 years. The route $R(I)$ of partial oxidation of ethylene (108) is always accompanied by the route R(II) of highly exothermic complete oxidation of ethylene (109):

(I) $\mathrm{C}_{2} \mathrm{H}_{4}+1 / 2 \mathrm{O}_{2}=\mathrm{C}_{2} \mathrm{H}_{4} \mathrm{O}+28 \mathrm{kcal} / \mathrm{mol}$

(II) $\mathrm{C}_{2} \mathrm{H}_{4}+3 \mathrm{O}_{2}=2 \mathrm{CO}_{2}+2 \mathrm{H}_{2} \mathrm{O}+316 \mathrm{kcal} / \mathrm{mol}$ (109)

The possible reaction mechanism is given in $(25)$. It is based on the presumption that ethylene participation in the oxidation is via Eley-Rideal mechanism. The selective oxidation is realized by the participation of adsorbed molecular oxygen, whereas the adsorbed atomic oxygen is responsible for the complete oxidation of ethylene.

$\mathrm{ZO}$ and $\mathrm{ZO}_{2}$ in (25) are $\mathrm{Ag}_{2} \mathrm{O}_{2}$ and $\mathrm{Ag}_{2} \mathrm{O}_{3}$, respectively, according to Ionov et al. [122], who assumes that $\mathrm{Z}$ is the lowest surface oxide of $\mathrm{Ag}_{2} \mathrm{O}$.

\subsubsection{In the absence of dichloroethane (DCE)}

The kinetic model based on mechanism (25) derived by applying Eq. (42) is given below [123]: 


$$
\begin{aligned}
\mathrm{R}(\mathrm{I}) & =\frac{k_{1} P_{\mathrm{O}_{2}} P_{\mathrm{e}}}{1+k_{3} P_{\mathrm{O}_{2}}+k_{4} P_{\mathrm{e}}} \\
\mathrm{R}(\mathrm{II}) & =\frac{k_{2} P_{\mathrm{O}_{2}} P_{\mathrm{e}}}{1+k_{3} P_{\mathrm{O}_{2}}+k_{4} P_{\mathrm{e}}}
\end{aligned}
$$

where $P_{\mathrm{O}_{2}}$ and $P_{\mathrm{e}}$ are the outlet partial pressures of $\mathrm{O}_{2}$ and $\mathrm{C}_{2} \mathrm{H}_{4}$, respectively. The Eqs. (110) and (111) can be used to describe the $\mathrm{C}_{2} \mathrm{H}_{4}$ oxidation rates at $\mathrm{CO}_{2}$ concentrations in reaction mixture below $10 \%$.

For the selectivity of the process, the following equation is developed:

$$
S=\frac{1}{0.83+\left(0.17+\frac{k_{2}}{k_{1}}\right) \cdot \frac{F_{\mathrm{e}}}{F_{\mathrm{O}_{2}}}}
$$

In this equation, two factors influencing the selectivity are included i.e. the kinetic term $k_{2} / k_{1}$ (from Eqs. (110) and (111)) and the feedstock composition through the $F \mathrm{e} / F_{\mathrm{O}_{2}}$ term. $F_{\mathrm{e}}$ and $F_{\mathrm{O}_{2}}$ are the flow rates of ethylene and oxygen at the entrance of the reactor.

\subsubsection{In the presence of DCE}

In industry, the process of ethylene oxidation is carried out in the presence of trace quantity of DCE from 0.2 to 28 ppm. The presence of selectivity promoter DCE changes the kinetic behavior of the reaction system. From the experimental data, the rate of complete oxidation $\mathrm{R}(\mathrm{II})^{\mathrm{DCE}}$ is inhibited to a greater extent than that of epoxidation $\mathrm{R}(\mathrm{I})^{\mathrm{DCE}}$. The developed model [124] for this case should take the presence of DCE in the reaction mixture into account.

$$
\begin{gathered}
\mathrm{R}(\mathrm{I})^{\mathrm{DCE}}=\frac{k_{1}^{\mathrm{DCE}} P_{\mathrm{O}_{2}} P_{\mathrm{e}}-k_{2}^{\mathrm{DCE}} P_{\mathrm{O}_{2}} P_{\mathrm{e}} P_{\mathrm{DHE}}^{k_{7}}}{1+k_{5}^{\mathrm{DCE}} P_{\mathrm{O}_{2}}+k_{6}^{\mathrm{DCE}} P_{\mathrm{e}}} \\
\mathrm{R}(\mathrm{II})^{\mathrm{DCE}}=\frac{k_{3}^{\mathrm{DCE}} P_{\mathrm{O}_{2}} P_{\mathrm{e}}-k_{4}^{\mathrm{DCE}} P_{\mathrm{O}_{2}} P_{\mathrm{e}} P_{\mathrm{DHE}}^{k_{8}}}{1+k_{5}^{\mathrm{DCE}} P_{\mathrm{O}_{2}}+k_{6}^{\mathrm{DCE}} P_{\mathrm{e}}}
\end{gathered}
$$

The influence of DCE used as promoter on reaction rates is reflected by the values of the kinetic constants compared with those values for the non-promoted catalyst (from Eqs. (110) and (111)) and by introducing terms acknowledging the influence of the content of selectivity promoter. The reaction orders with respect to DCE - $k_{7}$ and $k_{8}$ are dimensionless. Their values are $k_{7}=0.19$ and $k_{8}=0.07$ at all reaction temperatures. In the absence of the selectivity promoter $\left(P_{\mathrm{DCE}}=0\right)$, the DCE term becomes equal to zero and the kinetic equations have the same form as those for non-promoted catalysts (Eqs. (110) and (111)). The average deviations of the model for the partial oxidation and complete oxidation are $19 \%$ and $25 \%$, respectively.

It is a point of great practical importance to study the influence of different factors like promoter concentration, temperature, feed composition, and conversion degree of ethylene on the selectivity of the catalyst for ethylene oxide. The experiment results show that the selectivity does not change with the change of conversion degree of ethylene. The most important factor influencing the selectivity is found to be the concentration of the promoter DCE in the feed gas stream. The Eq. (115) describes the dependence of selectivity in the presence of DCE on different factors. $S^{\mathrm{DCE}}=$

$$
\frac{6\left(k_{1}^{\mathrm{DCE}}-k_{2}^{\mathrm{DCE}} P_{\mathrm{DCE}}^{0.19}\right)}{\left(5+\frac{F_{\mathrm{e}}}{F_{\mathrm{O}_{2}}}\right)\left(k_{1}^{\mathrm{DCE}}-k_{2}^{\mathrm{DCE}} P_{\mathrm{DCE}}^{0.19}\right)+\frac{6 F_{\mathrm{e}}}{F_{\mathrm{O}_{2}}}\left(k_{3}^{\mathrm{DCE}}-k_{4}^{\mathrm{DCE}} P_{\mathrm{DCE}}^{0.07}\right)}
$$

If the ethylene and the oxygen flow rates are equal, i.e. $F_{\mathrm{e}}$ $=F_{\mathrm{O}_{2}}$, then $\mathrm{Fe} / F_{\mathrm{O}_{2}}=1$ and Eq. (115) reduces to Eq. (116). The average deviation for the model selectivity according to Eq. (115) from the experimental data is $\pm 6 \%$.

$$
\begin{gathered}
S^{\mathrm{DCE}}=\frac{\mathrm{R}(1)^{\mathrm{DCE}}}{\mathrm{R}(1)^{\mathrm{DCE}}+\mathrm{R}(2)^{\mathrm{DCE}}}= \\
\frac{k_{1}^{\mathrm{DCE}}-k_{2}^{\mathrm{DCE}} P_{\mathrm{DCE}}^{0.19}}{\left(k_{1}^{\mathrm{DCE}}-k_{2}^{\mathrm{DCE}} P_{\mathrm{DCE}}^{0.19}\right)+\left(k_{3}^{\mathrm{DCE}}-k_{4}^{\mathrm{DCE}} P_{\mathrm{DCE}}^{0.07}\right)}
\end{gathered}
$$

The dependence of selectivity from DCE concentration might be approximated to:

$$
S=a \cdot C_{\mathrm{DCE}}+b
$$

The slope " $a$ " and the intercept " $b$ " depend on the reaction temperature and the feed composition. The physical meaning of " $b$ " is the selectivity in the absence of DCE:

$$
S=b \text { at } C_{\mathrm{DCE}}=0
$$

The physical meaning of " $a$ " is the sensitivity of the selectivity to DCE concentration.

$$
a_{1}=1-\frac{k_{2}}{k_{1}} P_{\mathrm{DCE}}^{0.19} \text { and } a_{2}=1-\frac{k_{4}}{k_{3}} P_{\mathrm{DCE}}^{0.07}
$$

where $a_{1}$ and $a_{2}$ are the sensitivities to the selectivities of the selective and total oxidation toward DCE.

In Table 3, data about the influence of DCE on the reaction rates following the reaction routes $\mathrm{R}(\mathrm{I})$ and $\mathrm{R}(\mathrm{II})$ are presented.

Table 3 Reaction route rates decrease at different DCE concentrations compared to rates measured without DCE presence

\begin{tabular}{rccccc}
\hline $\begin{array}{r}C_{\mathrm{DCE}} / \\
\mathrm{ppm}\end{array}$ & $T / \mathrm{K}$ & $P_{\mathrm{O} 2} / \mathrm{atm}$ & $P_{\mathrm{e}} / \mathrm{atm}$ & $\Delta \mathrm{R}(\mathrm{I}) / \%$ & $\Delta \mathrm{R}(\mathrm{II}) / \%$ \\
\hline 0.2 & 537 & 0.280 & 0.284 & 28.4 & 67.3 \\
2.0 & 537 & 0.383 & 0.143 & 43.5 & 78.6 \\
24.4 & 513 & 0.187 & 0.431 & 66.6 & 90.9 \\
\hline
\end{tabular}

From Eq. (119), we have calculated the critical concentration of DCE, at which the selective and total oxidation stop (Table 4).

The depressing combustion reaction takes place at lower promoter concentrations. This means that at $C_{\mathrm{DCE}}>$ $C_{\text {comb }}^{\text {crit }}$ and $C_{\mathrm{DCE}}<C_{\mathrm{epox}}^{\text {crit }}$ we might expect $100 \%$ selectivity 
Table 4 Critical concentrations of DCE

\begin{tabular}{lccc}
\hline \multirow{2}{*}{ Reaction } & \multicolumn{3}{c}{ DCE concentration $(\mathrm{ppm})$} \\
\cline { 2 - 4 } & $413 \mathrm{~K}$ & $537 \mathrm{~K}$ & $565 \mathrm{~K}$ \\
\hline Epoxidation & 208 & 160 & 120 \\
Combustion & 99 & 65 & 41 \\
\hline
\end{tabular}

but with very low degree of conversion.

\subsubsection{In the presence of $\mathrm{CO}_{2}$}

In some industrial units, the circulating gas for ethylene epoxidation contains a large amount of $\mathrm{CO}_{2}$. Our data showed that both the partial and the complete oxidation of $\mathrm{C}_{2} \mathrm{H}_{4}$ are inhibited to a considerable extent at $10 \%-30 \%$ $\mathrm{CO}_{2}$ concentration in the reaction mixture.

An explanation of the inhibiting effect of $\mathrm{CO}_{2}$ on the oxidation of $\mathrm{C}_{2} \mathrm{H}_{4}$ may be proposed based on the mechanism (25) with two additional steps.

$$
\begin{aligned}
& \text { 5) } \mathrm{ZO}+\mathrm{CO}_{2}=\mathrm{ZO} \cdot \mathrm{CO}_{2} \\
& \text { 6) } \mathrm{ZO}_{2}+\mathrm{CO}_{2}=\mathrm{ZO}_{2} \cdot \mathrm{CO}_{2}
\end{aligned}
$$

In view of the fact that both the partial and the complete oxidation are retarded in the presence of $\mathrm{CO}_{2}$, it is obvious that $\mathrm{CO}_{2}$ molecules are adsorbed on both active sites of $\mathrm{ZO}$ and $\mathrm{ZO}_{2}$.

The following model [125] is obtained from the scheme (25) with additional steps (120):

$$
\begin{aligned}
& \mathrm{R}(\mathrm{I}) \mathrm{CO}_{2}=\frac{k_{1}^{\mathrm{CO}} P_{\mathrm{O}_{2}} P_{\mathrm{e}}-k_{2}^{\mathrm{CO}_{2}} P_{\mathrm{O}_{2}} P_{\mathrm{e}} P_{\mathrm{CO}_{2}}}{1+k_{5}^{\mathrm{CO}_{2}} P_{\mathrm{O}_{2}}+k_{6}^{\mathrm{CO}} P_{\mathrm{e}}+k_{7}^{\mathrm{CO}_{2}} P_{\mathrm{CO}_{2}}} \\
& \mathrm{R}(\mathrm{II})^{\mathrm{CO}}=\frac{k_{3}^{\mathrm{CO}_{2}} P_{\mathrm{O}_{2}} P_{\mathrm{e}}-k_{4}^{\mathrm{CO}_{2}} P_{\mathrm{O}_{2}} P_{\mathrm{e}} P_{\mathrm{CO}_{2}}}{1+k_{5}^{\mathrm{CO}_{2}} P_{\mathrm{O}_{2}}+k_{6}^{\mathrm{CO}} P_{\mathrm{e}}+k_{7}^{\mathrm{CO}_{2}} P_{\mathrm{CO}_{2}}}
\end{aligned}
$$

The effect of $\mathrm{CO}_{2}$ concentrations on the reaction rates according to the reaction routes $\mathrm{R}(\mathrm{I})$ and $\mathrm{R}(\mathrm{II})$ is presented in Table 5.

Table 5 Rates decrease at different $\mathrm{CO}_{2}$ concentrations compared to rates measured without $\mathrm{CO}_{2}$ presence

\begin{tabular}{ccccc}
\hline$C_{\mathrm{CO} 2} / \%$ & $T / \mathrm{K}$ & $P_{\mathrm{O} 2} / P_{2}$ & $\Delta \mathrm{R}(1) / \%$ & $\Delta \mathrm{R}(2) / \%$ \\
\hline 12.5 & 513 & $2: 1$ & 55.0 & 81.0 \\
17.0 & 513 & $2: 1$ & 62.0 & 88.3 \\
25.0 & 513 & $2: 1$ & 70.0 & 93.5 \\
\hline
\end{tabular}

\subsubsection{Influence of water}

The presence of $0.216 \%$ or more $\mathrm{H}_{2} \mathrm{O}$ in the feed on the selective oxidation of $\mathrm{C}_{2} \mathrm{H}_{4}$ over silver catalyst at 210-264 ${ }^{\circ} \mathrm{C}$ inhibits the epoxidation of $\mathrm{C}_{2} \mathrm{H}_{4}$ to $\mathrm{C}_{2} \mathrm{H}_{4} \mathrm{O}$ [126]. The effect grows stronger upon increasing the temperature from 210 to $264{ }^{\circ} \mathrm{C}$. At the same time, it promotes the combustion of $\mathrm{C}_{2} \mathrm{H}_{4}$. This effect passes through a maximum at 240 ${ }^{\circ} \mathrm{C}$ upon increasing the temperature from 210 to $264{ }^{\circ} \mathrm{C}$.

The net effect of the presence of $\mathrm{H}_{2} \mathrm{O}$ in the feed is to in- crease the $\mathrm{C}_{2} \mathrm{H}_{4}$ oxidation degree, as the promoting effect on the combustion is stronger than the inhibiting effect on the epoxidation. The promoting effect dominates over the inhibiting effect throughout the whole studied temperature interval $\left(210-264^{\circ} \mathrm{C}\right)$.

The selectivity of our catalyst for $\mathrm{C}_{2} \mathrm{H}_{4} \mathrm{O}$ deteriorates in the presence of $\mathrm{H}_{2} \mathrm{O}$ in the feed in contradiction to the data reported by other authors, in which either selectivity increase or no influence of $\mathrm{H}_{2} \mathrm{O}$ on the selectivity.

\subsubsection{Kinetics of ethylene oxide oxidation}

During the total oxidation of ethylene oxide at catalyst bed temperature above $300{ }^{\circ} \mathrm{C}$, the reaction displays a tendency to pass over to an autothermic regime [127-129]. Under such conditions in the temperature range between $310-335{ }^{\circ} \mathrm{C}$ the thermooscillations were observed. The form of the oscillations is comparatively simple. With some minor exceptions, it can be approximated to a regular sinusoid. The period of the oscillations varies from 7 to $38 \mathrm{~min}$, while the amplitude varies from $3 \%$ to $19 \%$ of the average temperature value. The oscillatory period and the amplitude change with the feed composition and the contact time independently to each other.

The possible explanation of the oscillation behavior is based on periodically coverage of silver surface by adsorbed molecular oxygen, followed by coverage by adsorbed atomic oxygen, which have different activity, but both forms participate in the complete oxidation of ethylene oxide.

Observed autothermic oscillations may lead to spontaneous explosions which happens in the industrial reactors due to the very high linear velocity of the reactants through catalysts bed, erosion of the supported silver takes place. This silver is collected at the exit of the reactor where temperature is relatively high and it further catalyzes the complete oxidation of ethylene oxide and all other organic compound in the reaction mixture.

For this reason, the kinetics of the complete oxidation of ethylene oxide over a supported silver catalyst was studied at the temperature range $100-300{ }^{\circ} \mathrm{C}$ at atmospheric pressure. The ethylene oxide oxidation starts at $190{ }^{\circ} \mathrm{C}$. The kinetics of the total oxidation of ethylene oxide on silver catalyst was studied in the absence and presence of DCE.

$$
r^{\mathrm{EO}}=\frac{k_{1}^{\mathrm{EO}} P_{\mathrm{O}_{2}} P_{\mathrm{e}}-k_{2}^{\mathrm{EO}} P_{\mathrm{O}_{2}} P_{e} P_{\mathrm{DCE}}^{k_{7}}}{\left(1+k_{3}^{\mathrm{EO}} P_{\mathrm{O}_{2}}+k_{4}^{\mathrm{EO}} P_{\mathrm{O}_{2}}^{0.5}+k_{5}^{\mathrm{EO}} P_{\mathrm{EO}}+k_{6}^{\mathrm{EO}} P_{\mathrm{EO}} P_{\mathrm{O}_{2}}^{-0.5}\right)^{2}}
$$

The obtained model (123) describes the reaction rate of total ethylene oxide (EO) oxidation in the presence of DCE. The reaction order for DCE, $k_{7}$, has a constant value, which does not change with changes of the temperature.

\subsubsection{Modeling of the industrial reactors for ethylene}




\section{epoxidation}

The models presented above allow the designer of industrial catalysts to control precisely the catalytic process at optimal conditions. This is especially important for controlling the concentration of DCE in reaction mixture. The kinetic models for the process of ethylene epoxidation were built using the reaction mechanism proposed based on the extended knowledge about the studied reaction system and used catalyst. For this reason, they describe the features of the system quite well and were used for dynamic modeling of industrial ethylene oxide reactor [130]. The authors accepted that two reaction routes (see (108) and (109)) take place.

The kinetic model of the reactions in the presence of DCE (Eqs. (113) and (114)) was incorporated into the multi-tubular reactor model. The values of the kinetic parameters were slightly adjusted according to the used catalyst. The heterogeneous reactor model was built underlying the major assumptions:

- The gas properties follow the ideal gas law;

- The heat of reaction and gas heat capacities are considered constant;

- The packed bed is uniformly packed in tubes of small diameter with negligible wall effect;

- The radiation effects between solid catalyst and the gas are considered negligible;

- The resistance to heat and mass transfer in the catalyst pellets is negligible;

- The rate of reaction is uniform throughout the particle and the reaction occurs on the solid surface.

The reactor model was compared and validated against the industrial EO reactor and it could predict plant data well. In Table 6, it is presented the simulation results and validation of the industrial reactor data.

Other authors [131] have developed a dynamic model of industrial EO reactor aiming at studying the catalyst deactivation and its optimization. They used the three-route reaction scheme proceeded in the presence of $\mathrm{DCH}$. The third

Table 6 Comparison between modeling of industrial reactor for EO production and plant data

\begin{tabular}{lcc}
\hline \multirow{2}{*}{ Property } & \multicolumn{2}{c}{ Condition at reactor outlet } \\
\cline { 2 - 3 } & Simulation & Plant \\
\hline Ethylene & 0.3705 & 0.3743 \\
Oxygen & 0.0359 & 0.0358 \\
EO & 0.0369 & 0.0386 \\
$\mathrm{CO}_{2}$ & 0.0419 & 0.0420 \\
Temperature & $268{ }^{\circ} \mathrm{C}$ & $268{ }^{\circ} \mathrm{C}$ \\
Pressure & $13.8 \mathrm{bar}$ & $13.9 \mathrm{bar}$ \\
Selectivity & $86.5 \%$ & $87.23 \%$ \\
\hline
\end{tabular}

Gas composition in mol parts. reaction route is total oxidation of EO.

$$
\begin{aligned}
& \mathrm{R}(\mathrm{I}): \mathrm{C}_{2} \mathrm{H}_{4}+1 / 2 \mathrm{O}_{2}=\mathrm{C}_{2} \mathrm{H}_{4} \mathrm{O} \\
& \mathrm{R}(\mathrm{II}): \mathrm{C}_{2} \mathrm{H}_{4}+3 \mathrm{O}_{2}=2 \mathrm{CO}_{2}+2 \mathrm{H}_{2} \mathrm{O} \\
& \text { R(III): } \mathrm{C}_{2} \mathrm{H}_{4} \mathrm{O}+5 / 2 \mathrm{O}_{2}=2 \mathrm{CO}_{2}+2 \mathrm{H}_{2} \mathrm{O}
\end{aligned}
$$

The kinetic model also consists of three reaction rate equations:

$$
\begin{gathered}
\mathrm{R}(\mathrm{I})^{\mathrm{DCE}}=\frac{k_{1}^{\mathrm{DCE}} P_{\mathrm{O}_{2}} P_{\mathrm{e}}-k_{2}^{\mathrm{DCE}} P_{\mathrm{O}_{2}} P_{\mathrm{e}} P_{\mathrm{DHE}}^{k_{7}}}{1+k_{5}^{\mathrm{DCE}} P_{\mathrm{O}_{2}}+k_{6}^{\mathrm{DCE}} P_{\mathrm{e}}} \\
\mathrm{R}(\mathrm{II})^{\mathrm{DCE}}=\frac{k_{3}^{\mathrm{DCE}} P_{\mathrm{O}_{2}} P_{\mathrm{e}}-k_{4}^{\mathrm{DCE}} P_{\mathrm{O}_{2}} P_{\mathrm{e}} P_{\mathrm{DHE}}^{k_{8}}}{1+k_{5}^{\mathrm{DCE}} P_{\mathrm{O}_{2}}+k_{6}^{\mathrm{DCE}} P_{\mathrm{e}}} \\
\mathrm{R}(\mathrm{III})^{\mathrm{DCE}}=\frac{k_{1}^{\mathrm{EO}} P_{\mathrm{O}_{2}} P_{\mathrm{e}}-k_{2}^{\mathrm{EO}} P_{\mathrm{O}_{2}} P_{e} P_{\mathrm{DCE}}^{k_{7}}}{\left(1+k_{3}^{\mathrm{EO}} P_{\mathrm{O}_{2}}+k_{4}^{\mathrm{EO}} P_{\mathrm{O}_{2}}^{0.5}+k_{5}^{\mathrm{EO}} P_{\mathrm{EO}}+k_{6}^{\mathrm{EO}} P_{\mathrm{EO}} P_{\mathrm{O}_{2}}^{-0.5}\right)^{2}}
\end{gathered}
$$

The reactor model predictions were validated against the data from an industrial EO reactor for 3 years period. The work rate (tones/day) and the selectivity have maximum $13 \%$ and $6 \%$ difference between the model prediction and the plant data, respectively. Therefore, the model predicts the plant data and reactor behavior well. These results were used for online optimizing of reactor performance with aim of obtaining the maximal selectivity at daily production of ethylene oxide between 110 and 120 tone/day.

\section{Conclusions}

It does not mean that an optimum industrial catalyst has already been created by selecting the optimum active substance. Undoubtedly, chemical composition is the main factor that determines the catalytic properties. However, even for the same chemical composition, depending on the conditions and methods of preparation, the catalytic properties of the catalyst may be varied within a considerable interval. This is a consequence of changes in the nature of interaction between catalyst components, catalyst dispersion, porous and crystal structure, and other factors which have an essential influence on the catalytic reactions. In many cases, the physical mechanical factors have been proved to be decisive in selecting a certain catalyst production technology and a given type of catalysts.

Detailed study of the kinetics is expensive and time-consuming process and should be done on the catalysts that have already passed other chemical, physical, mechanical, and physical-chemical tests. Studies of the catalyst deactivation are a very important part of the process of building kinetic models. Heat and mass transfer processes always accompany heterogeneous catalytic reactions and it is very important to evaluate properly the heat and mass transfer effect in a sudied catalytic reaction. Reliable and precise data can be obtained only if the laboratory catalytic reactors are properly selected and the analytical methods 
used are fast, precise, and reliable. The reaction rate is the only precise and reliable measure for the catalytic activity.

Interpretation of the results from kinetic studies should always be done with considering the results obtained from the application of other characterization techniques chemical, physical, morphological, and mechanical. In this sense it is very important that there is the opportunity to combine catalytic activity measurements with applying some "in situ" physical methods by which the additional information about the catalyst changes during the catalytic runs can be obtained.

In general, the catalyst should be regarded as an inseparable part in the larger system consisting of the reaction medium and the catalyst itself. Heterogeneous catalysts are very complex systems with pre-programmed properties. Some of these properties do not depend very much on the conditions under which they were measured. However, in many cases the situation is different. During the course of catalyst operation in industrial reactors at high temperature and pressure, under the influence of the reaction media and other factors the catalyst structure, composition, and properties are changing. In some cases, these changes are small, but sometimes they are quite substantial.

As stated above, the application of industrial catalytic processes is of strategic importance for any developed country and without doubt, it belongs to the category of the so-called 'high tech' industries. It should be emphasized that without intensive research and development activities it is impossible to reach and maintain a high level of industrial innovation. In view of the above considerations, industry should play the leading role, which is especially important. As one of the chief employers and main consumer of research products, it is expected that industry will be the main initiator that will understand and define existing and future problems and find their solution. It has to be the provider of funding for R\&D teams.

The progress of chemical industry depends on its attitude toward chemical science and interest in research work, while the good shape of science is determined by the fact how far it turns its face to the demand and prospects for developments in industry.

\section{Acknowledgements}

This work was carried out as a part of activities of the SABIC Chair in Catalysis at King Abdulaziz University, Jeddah. The authors are thankful to SABIC for financial support of the Chair.

\section{References}

1 Petrov L. In: Petrov L, Bonev Ch eds. Catalysis for Sustain- able Chemistry: Structure, Processes and Industrial Applications. Proc UNIDO-ICS Workshop, Sofia, 3-6 November 2000, International Centre for Science and High Technology, United Nations Industrial Development Organization (UNIDO), Institute of Catalysis, Bulgarian Academy of Sciences, Sofia, 2001. 1

2 Kiperman S L. Fundamentals of Chemical Kinetics in Heterogeneous Catalysis. Moscow: Chemistry Publishing House, 1979

3 Temkin M I. Adv Catal, 1979, 28: 173

4 Murzin D, Salmi T. Catalytic Kinetics. Amsterdam: Elsevier, 2005

5 Vannice M. Albert Kinetics of Catalytic Reactions. Berlin: Springer, 2006

6 Bond G C. Heterogeneous Catalysis and Applications. 2nd Ed. Oxford: Oxford University Press, 1987

7 Froment G F, Bischoff K B. Chemical Reactor Analysis and Design. New York: Willey, 1990

8 Somorjai G A. Principles of Surface Chemistry and Catalysis. New York: Willey, 1994

9 Thomas J M, Thomas W J. Principles and Practice of Heterogeneous Catalysis. Weinheim: VCH, 1996

10 Chokendorf I, Niemantsverdriet J W. Concept of Modern Catalysis and Kinetics. Weinheim: VCH, 2003

11 Krylov O V. Heterogeneous Catalysis. Moscow: Akademkniga, 2004

12 Bastiansen O. The Law of Mass Action, A Centenary Volume 1864-1964. Oslo: Universitetsforlaget, 1964. 194

13 Langmuir I. J Am Chem Soc, 1916, 38: 2217

14 Langmuir I. J Am Chem Soc, 1918, 40: 1361

15 Boudart M, Djega-Mariadassou G. Kinetics of Heterogeneous Catalytic reactions. Princeton: Princeton University Press, 1984

16 Tolman R. Principles of Statistical Mechanics. Oxford: Clarendon Press, 1938

17 Evans M G, Polanyi M. Trans Faraday Soc, 1938, 34: 11

18 Semenov N N. Some Problems in Chemical Kinetics and Reactivity. London: Pergamon, 1958

19 Brønsted J N, Pedersen K. Z Physik Chem, 1924, 108: 185

20 Brønsted J N, LaMer V K. J Am Chem Soc, 1924, 46: 555

21 Temkin M I. Zhur Fiz Khim, 1941, 15: 296

22 Horiuti J. Adv Catal, 1957, 9: 339

23 Horiuti J, Nakamura T. Z Phys Chem (Frankfurt am Main), 1957, 11: 358

24 Horiuti J. Z Phys Chem (Frankfurt am Main), 1957, 12: 351

25 Horiuti J. J Catal, 1962, 1: 199

26 Temkin M I. Nauchnye Osnovy Podbora i Proizvodstva Katalizatorov. Novosibirsk: Siberian Branch of the Acad Sci USSR, 1964. 46

27 Bodenstein M. Z Phys Chem B, 1913, 85: 329

28 Frank-Kamenetzkii D A. Zh Phis Khim, 1940, 14: 695

29 Hougen O A, Watson K M. Chemical Process Principles Part 3

Kinetics and Catalysis. New York: Wiley, 1947

30 Temkin M I. Dokl Akad Nauk USSR, 1965, 161: 160

31 Temkin M I. Zhur Fiz Khim, 1957, 31: 3 
32 Temkin M I. Dokl Akad Nauk USSR, 1963, 152: 156

33 Temkin M I. J Res Inst Catal Hokaido University, 1968, 16: 355

34 King L E, Altman C. J Phys Chem, 1956, 60: 1375

35 Volkenstein M V, Goldshte B N. Dokl Acad Nauk SSSR, 1966, 170: 963

36 Temkin M I. Dokl Akad Nauk USSR, 1965, 165: 615

37 Petrov L A. In: Bonchev D, Rouvray D eds. Chemical Graph

Theory: Reactivity and Kinetics. London: Gordon and Breach

Science Publishers Ltd, 1992. 2

38 Christoffel E G. Catal Rev-Sci Eng, 1982, 24: 159

39 Temkin M I, Kiperman S L, Lukyanova L I. Dokl Acad Nauk SSSR, 1950, 74: 763

40 Berty J M. Catal Rev-Sci Eng, 1979, 20: 75

41 Berty J M. In: Leach B E ed. Applied Industrial Catalysis. Vol

1. New York: Academic Press, 1983

42 Wheeler A. Adv Catal, 1950, 3: 249

43 Frank-Kamenetzkii D A. Diffusion and Heat Transfer in Chemical Kinetics. Moscow: Nauka, 1987

44 Satterfield C N. Mass Transfer in Heterogeneous Catalysis. Cambridge: MIT Press, 1970

45 Aris R. The Mathematical Theory of Diffusion and Reaction in Permeable Catalysts I The Theory of Steady State. Oxford: Clarendon Press, 1970

46 Carberry J J. Catal Sci Technol, 1987, 8: 131

47 Haynes,Jr H W. Catal Rev-Sci Eng, 1988, 30: 563

48 Park I-S, Do D D, Rodrigues A E. Catal Rev-Sci Eng, 1996, 38: 189

49 Ma Y H, Mancel C. Adv Chem Ser, 1973, 121: 392

50 Weisz P B, Prater C D. Adv Catal, 1954, 6: 143

51 Hughes R. Deactivation of Catalysts. New York: Academic Press, 1984

52 Krishna A S. Catal Rev-Sci Eng, 1990, 32: 279

53 Oudar J, Wise H. Deactivation and Poisoning of Catalysts. New York: Marcel Dekker, 1985

54 Butt J B, Petersen E E. Activation, Deactivation and Poisoning of Catalysts. San Diego: Academic Press, 1988

55 Hegedus L I, McCabe R W. Catalyst Poisoning. New York: Marcel Dekker, 1984

56 Bartholomew C H. Appl Catal A, 2001, 212: 17

57 Kumbilieva K, Petrov L, Alhamed Y, Al-Zahrani A. Chin J Catal, 2011, 32: 387

58 Box G E P, Hill W J. Technometrics, 1967, 9: 57

59 Kittrell J R. Adv Chem Eng, 1970, 8: 97

60 Petrov L, Eliyas A, Maximov Ch. Ind Eng Chem Res, 1991, 30: 639

61 Spivak S I, Timoshenko V I, Slinko M G. Dokl Acad Nauk SSSR, 1970, 192: 580

62 Slinko M G, Spivak S I, Timoshenko V I. Kinet Katal, 1972, 13: 1570

63 Spivak S I, Slinko M G, Timoshenko V I. React Kinet Catal Lett, 1974, 1: 99

64 Petrov L A, Shopov D M. React Kinet Catal Lett, 1977, 7: 261

65 Nelder J A, Mead R. Computer J, 1965, 7: 308
66 Marquard D. J Soc Indust Appl Math, 1963, 11

67 Rosenbrock H H, Storey C. Computational Techniques for Chemical Engineering. New York: Wiley, 1968

68 Akhmadishin Z Sh, Spivak S I, Shmelev A S. React Kinet Catal Lett, 1981, 16: 7

69 Gennari F, Seneci P, Miertus S. Catal Rev-Sci Eng, 2000, 42 385

70 Farrusseng D. Surf Sci Reports, 2008, 63: 487

71 Rodemerk U, Linke D. In: de Jong K P ed. Synthesis of Solid Catalysts. Weinheim: Wiley-VCH Verlag, 2009. 217

72 Petrov L. NATO Science Series, II Mathematics, Physics and Chemistry, 2002, 69: 13

73 Bobrov N N, Parmon V N. NATO Science Series, II Mathematics, Physics and Chemistry, 2002, 69: 197

74 Boudart M. Adv Catal, 1969, 20: 153

75 Parmon V N. Doklady Phys Chem, 2007, 413: 42

76 Parmon V N. Thermodynamics of Non-equilibrium Processes with a Particular Application to Catalysis. Amsterdam: Elsevier, 2010

77 Murzin D Yu. Chem Eng Sci, 2009, 64: 1046

78 Murzin D Yu. J Mol Catal A, 2010, 315: 226

79 Murzin D Yu. J Catal, 2010, 276: 85

80 Hvolbæk B, Janssens $\mathrm{T}$ V W, Clausen B S, Falsig H, Christensen C H, Nørskov J K. Nanotoday, 2007, 2: 14

81 Kolasinki K W. Surface Science. Fundamentals of Catalysis and Nanoscience. Chichester: Wiley, 2002

82 Temkin M I. Kinet Katal, 1984, 25: 299

83 Boudart M, Tamaru K. Catal Lett, 1991, 9: 15

84 van Santen R. In: Cybulski A, Moulijn J A, Stankiewcz A eds. Novel Concepts in Catalysis and Chemical Reactors: Improving the Efficiency for the Fyuture. Weinhem: Wiley, 2010. 1

85 Kumbilieva K, Petrov L. Chin J Catal, 2011, 32: 51

86 Kumbilieva K, Petrov L. Catal Ind, 2011, 3: 748

87 Kumbilieva K, Petrov L, Gaidai N, Eliyas A, Nekrasov N, Lapidus L. Nanosci Nanotechnol, 2009, 9: 24

88 Eliyas A, Kumbilieva K, Petrov L. Nanosci Nanotechnol, 2009, 9: 50

89 Slinko M M, Jaeger N I. Stud Surf Sci Catal, 1994, 86: 47

90 Koleva M K, Petrov L A. In: Linke A N ed. Trends in Chemical Physics Research. New York: Nova Science Publishers, 2005. 167

91 Koleva M, Eliyas A, Petrov L. NATO Science Series, Series C: Mathematical and Physical Sciences, 2000, 546: 353

92 Berger R J, Kapteijn F, Moulijn J A, Marin G B, De Wilde J, Olea M, Chen D, Holmen A, Lietti L, Tronconi E, Schurman Y. Appl Catal A, 2008, 342: 3

93 Matros Yu Sh. Nonsteady State Processes in Catalytic Reactors. Moscow: Nauka, 1982

94 Kraushaar-Czarnetzki B, Muller S P. In: de Jong K P ed. Synthesis of Solid Catalysts. Weinheim: Wiley-VCH Verlag, 2009. 173

95 Schneider P, Smith J M. AIChE J, 1968, 14: 762

96 Haynes H W, Jr, Sharma P N. AIChE J, 1973, 19: 1043

97 Ma Y H, Mancel C. Adv Chem Ser, 1973, 121: 392

98 Hashimoto N, Smith J M. Ind Eng Chem Fundam, 1973, 12: 
353

99 Solcova O, Soukup K, Schneider P. Microporous Mesoporous Mater, 2006, 91: 100

100 Kubin M. Collec Czech Chem Commun, 1965, 30: 1104

101 Kubin M. Collec Czech Chem Commun, 1965, 30: 2900

102 Kucera E. J Chromat, 1965, 19: 237

103 Wicke E, Kallenbach R. Kolloid Z, 1941, 17: 135

104 Gubilaro L G, Gioria F, Grego G Jr. Chem Eng J, 1970, 1: 85

105 Dogu G, Smith J M. AIChE J, 1975, 21: 58

106 Dogu G, Smith J M. Chem Eng Sci, 1976, 31: 123

107 Mofat A J. J Catal, 1978, 54: 107

108 Hou K, Fowles M, Hughes R. Chem Eng Res Des, 1999, 77: 55

109 Stary T, Solcova O, Schneider P, Marek M. Chem Eng Sci, 2006, 61: 5934

110 Soukup K, Schneider P, Solcova O. Chem Eng Sci, 2008, 63: 1003

111 Solcova O, Snajdaufova H, Schneider P. Chem Eng Sci, 2001, 56: 5231

112 Shen L H, Chen Z X. Chem Eng Sci, 2007, 62: 3748

113 Satterfield C N. Mass Transfer in Heterogeneous Catalysis, IT Press, 1970

114 Wang CT, Smith J M. AIChE J, 1983, 29: 132

115 Hoogschagen J. Ind Eng Chem, 1955, 47: 906

116 Scott DS, Dullien F A L. AIChE J, 1962, 8: 113

117 Diesler P F, Wilhelm R H. Ind Eng Chem, 1953, 45: 1219
118 Petrov L A, Kirkov N V, Shopov D M. Kinet Katal, 1985, 26: 897

119 Petrov L, Kumbilieva K, Kirkov N. Appl Catal, 1990, 59: 31

120 Petrov L, Vladov Ch, Neshev N, Bonev Ch, Prahov L, Kirkov N, Vasileva M, Filkova D, Dancheva S. Bulgarian Patent 41960, 1986

121 Petrov L, Vladov Ch, Bonev Ch, Prahov L, Kirkov N, Eliyas A, Neshev N, Filkova D, Dancheva S, Geus J W. In: EuropaCat-2, Book of Abstracts. Maastricht, The Netherlands, 1995. 646

122 Ionov Y V, Temkina E M, Kamensky D, Kulkova N V, Temkin M I. Kinet Katal, 1980, 21: 1269

123 Petrov L, Eliyas A, Shopov D. Appl Catal, 1985, 18: 87

124 Petrov L, Eliyas A, Shopov D. Appl Catal, 1986, 24: 145

125 Eliyas A, Petrov L. Appl Catal, 1990, 62: 11

126 Liu W, Eliyas A, Petrov L. Appl Catal, 1990, 61: 265

127 Petrov L, Eliyas A, Maximov Ch, Shopov D. Appl Catal, 1988, 41: 23

128 Eliyas A, Petrov L, Shopov D. Appl Catal, 1988, 41: 39

129 Petrov L, Eliyas A, Maximov Ch. Ind Eng Chem Res, 1991, 30: 639

130 Cornelio A A. Indian Chem Eng, 2006, 48: 164

131 Ghaffari S, Ngian K, Romagnoli J, Gomes V G. CHISA-2006, 17th International Congress of Chemical and Process Engineering. 2006. 9 